\title{
Model of $\mathrm{BaTiO}_{3}$ Synthesis in Three-Zone Reactor Accounting for Thermal and Concentration Expansion Effect
}

\author{
A.A. Markov \\ Ishlinsky Institute for Problems in Mechanics Russian Academy of Sciences (IPMech RAS), \\ Moscow, 119526, Russia \\ markov.ipm@yandex.ru
}

\begin{abstract}
This paper reports about a generalization of two-temperature model on study of thermal and mass dispersion and expansion in carbon combustion synthesis of oxides (CCSO) in porous media. The governing system of equations includes the Navier-Stokes equations for the gas phase and the thermoelasticity equations using the Duhamel-Neumann relations. The equations of conservation of mass and heat balance of the gas phase contain tensors of mass and thermal dispersion, conductive and convective transfer. The results of studying the effect of thermal and concentration expansion on variable porosity in the process of synthesis of micron barium titanate powders during carbon combustion are presented. A dimensionless form of the constitutive equations with similarity parameters is given. Dimensionless equations are applied to the numerical simulation of the effect of thermal and mass dispersion at variable porosity on the synthesis of micron barium titanate particles in a three-zone reactor. The results of calculations, including the effect of dispersion, pore tortuosity coefficient, particle diameter, local values of Peclet numbers are presented. The proposed model makes it possible to analyze and evaluate the effect of thermal and concentration expansion on the velocity of the combustion and synthesis front, as well as on the uniformity of the distribution of the reactants and the synthesis product in the reactor.
\end{abstract}

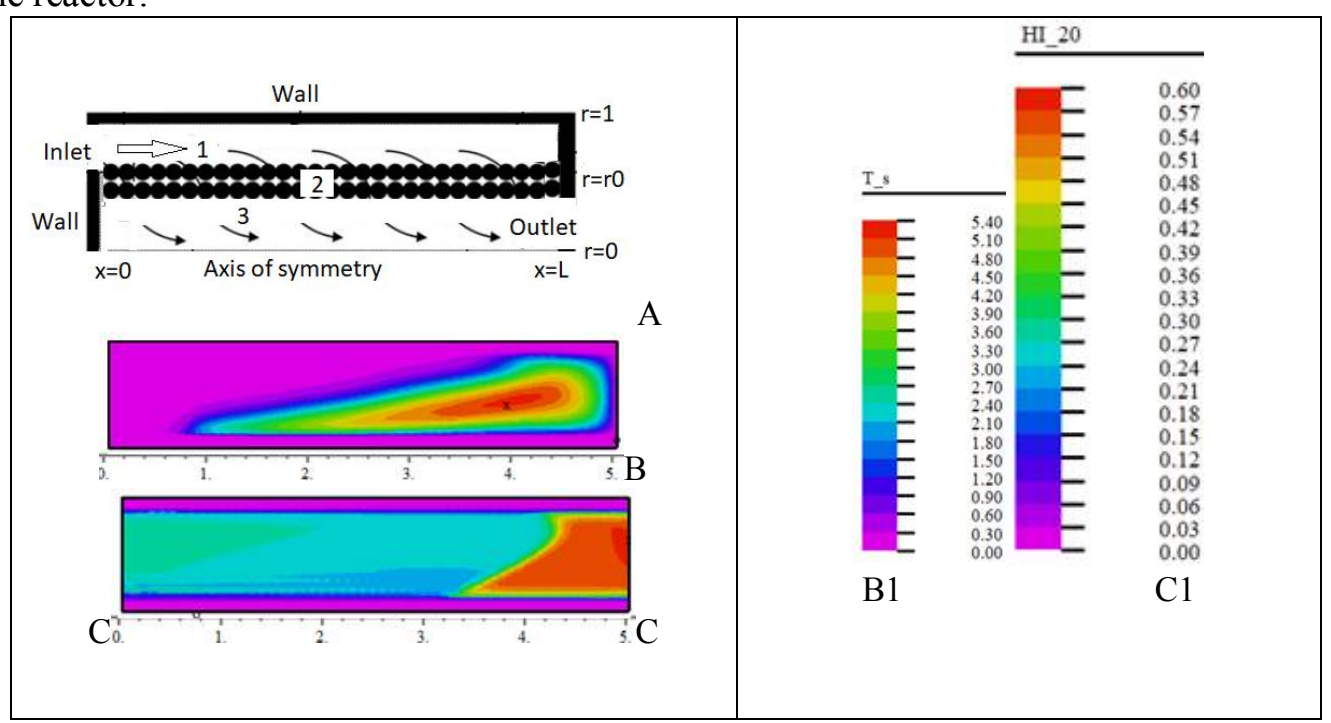

The schematic presentation of the combustion model fig. A. The simulation in the region $0<x<L, 0<r<1$ consisting of zone 1: $0<x<L, 0<r<R_{1}$, zone 2: $0<x<L, R_{2}<r<R_{1}$ and zone 3: $0<x<L, R_{1}<r<1$ is presented. The mixture of gas species $\mathrm{O}_{2}$ and $\mathrm{N}_{2}$ is supplied in zone 3 from the left section $x=0, R_{2}<r<1$. The gas mixture of $\mathrm{O}_{2}, \mathrm{~N}_{2}$ and $\mathrm{CO}_{2}$ is flown out in zone 1 from the boundary $x=L, 0<r<R_{1}$. Zone 2: shows the porous region of a mixture of carbon powders, reagents and synthesis products. Figures B and B1 present distribution and temperature of the solid phase at the time instant $t=0.88$. Figures $\mathrm{C}$ and $\mathrm{C} 1$ show the distribution of porosity at $t=0.88$ 


\title{
О модели синтеза титаната бария в трех-зонном реакторе с эффектами теплового и концентрационного расширения
}

\author{
А.А. Марков \\ Институт проблем механики им. А. Ю. Иилинского Российской академии наук \\ Россия, Москва, 119526, проспект Вернадского, д. 101-1 \\ markov.ipm@yandex.ru
}

\begin{abstract}
Аннотация
Исследование направлено на развитие двухтемпературной модели с целью изучения эффектов теплового и концентрационного расширения при синтезе сложных оксидов методом горения углерода CCSO. Определяющая система уравнений включает уравнения Навье-Стокса для газовой фазы и уравнения термоупругости с использованием соотношений Дюамеля - Неймана. Уравнения сохранения массы и теплового баланса газовой фазы содержат тензоры массовой и тепловой дисперсии, кондуктивного и конвективного переноса. Излагаются результаты исследования воздействия теплового и концентрационного расширения на переменную пористость в процессе синтеза микронных порошков титаната бария при горении углерода. Приведены результаты расчетов, включающие воздействие дисперсии, коэффициента извилистости пор, диаметра частиц, локальных величин чисел Пекле. Предлагаемая модель позволяет анализировать и оценивать влияние теплового и концентрационного расширения на скорость фронта горения и синтеза, а также на однородность распределения в реакторе реагентов и продукта синтеза.
\end{abstract}

Ключевые слова: переменная пористость, синтез горением углерода титаната бария в трехзонном реакторе, двухтемпературная модель.

\section{1. Введение}

Метод синтеза оксидов при горении углерода (CCSO) [1] стимулировал теоретические исследования [2-10]. Были предложены модели усреднения на основе взаимно-проникающих континуумов, в которых утраченная детальная информация о микро-масштабах, таких как конфигурация межфазных границ и т.п., присутствует в виде коэффициентов тепло- и массопереноса. В ряде работ показана важность дисперсионных слагаемых в усредненных уравнениях наряду с молекулярной диффузией тепла и вещества. Концентрационная и тепловая дисперсия вызывается флуктуациями массового и теплового потока, в то время как причиной диффузии является молекулярное движение. Различные модели тепловой дисперсии изучены в работах [11-17].

Моделирование синтеза материалов развивалось в работах [2-10], в приближении постоянных размеров пор, заданных априори. Следует заметить, что размеры пор, как правило, неравномерно распределены в реакторе синтеза и меняются во времени, влияя на потоки тепла, вещества реагентов и продуктов через пористую среду. Градиенты температуры и концентраций приводят к изменению объема и появлению перемещений и напряжений в твердой фазе. В работах $[18,19]$ рассмотрены модели спекания и синтеза порошковых смесей твердой фазы, с учетом взаимовлияния процессов объемных изменений в процессе спекания. В работе [20] проведены экспериментальные исследования спекания частиц феррита кобальта и титаната бария в процессе горения углерода 
Темой данного исследования является обобщение модели [7] для расчета эффектов переменной пористости при синтезе методом CCSO мелкодисперсных порошков. Исследуется влияние объемных изменений при синтезе титаната бария $\mathrm{BaTiO}_{3}$ на основе двухфазной модели тепловой и массовой дисперсии [7-10]. В связи с требованиями промышленного производства керамики на основе $\mathrm{BaTiO}_{3}$ актуальной проблемой является сбалансированное потребление энергии, продолжительности синтеза и качества конечного продукта, такого как форма и распределение частиц. Кинетика синтеза титаната бария рассмотрена в $[1,21,22]$. Основной целью предлагаемого моделирования является исследование концентрационного расширения твердой фазы в условиях межфазового тепло- и массобмена. Данное исследование опирается на результаты [18]. Предлагается теоретическая модель, позволяющая предсказывать характеристики волны горения при переменной пористости образца, когда происходит поверхностный тип горения. Проведено моделирование синтеза титаната бария методом CCSO в осесимметричном трехзонном реакторе [10], аналогичный плоской конфигурации дизельного сажевого фильтра [23]. Рассмотрены тепловые эффекты кинетики синтеза на основе термодинамических данных [24]. Предлагается формула нестационарного изменения пористости в зоне синтеза при заданном распределении пористости в начальный момент времени. Изучено влияние переменной пористости на процессы синтеза микронных частиц при тепловой и массовой дисперсии, сопутствующей конвективному и кондуктивному тепломассопереносу. Концентрационное расширение газовой фазы вследствие давления газа на поверхность пор замедляет уменьшение пор в процессе горения вследствие теплового и концентрационного расширения твердой фазы. Химические превращения происходят с изменением объема и сопровождаются появлением механических напряжений и деформаций дополнительно к напряжениям и деформациям вследствие высоких градиентов температуры.

Полученные результаты показывают существенное влияние объемных изменений в газе и твердой фазе. Предлагаемая модель позволяет анализировать воздействие теплового и концентрационного расширения на скорость синтеза и распределение продукта в зоне синтеза. В данной работе представлены результаты моделирования CCSO при размерах частиц реагентов и продуктов, превышающих микрон. Отметим, что при микронных размерах синтезируемых частиц характерные величины чисел Кнудсена малы и эффекты скольжения и скачков температуры [25] пренебрежимо малы. Однако, для субмикронных размеров частиц и пор становится существенным влияние слоев Кнудсена в газе около поверхности пор и необходим учет эффектов скольжения [26, 27].

\section{2. Теоретический анализ}

\section{1. Термодинамические данные химических реакций}

Локальное изменение свободной энергии Гиббса в зависимости от температуры определяется соотношениями [28]

$$
\begin{gathered}
\Delta G_{j}(T)=\Delta h_{j}(T)-T \Delta S_{j}(T), \\
\Delta h_{j}(T)=\Delta h_{j}(298)+\int_{298}^{T} c_{j p}(\varsigma) \mathrm{d} \varsigma,
\end{gathered}
$$

где $j=1, \ldots, 6$ - номера компонент $\mathrm{O}_{2}, \mathrm{CO}_{2}, \mathrm{C}, \mathrm{BaCO}_{3}, \mathrm{TiO}_{2}, \mathrm{BaTiO}_{3}$ соответственно; $\Delta h_{j}(298)$ - энтальпия компонента при температуре $298 \mathrm{~K}$ и $c_{j p}(T)$ - теплоемкость;

$$
c_{j p}(T)=a_{j}+b_{j} 10^{-3} T-c_{j} 10^{5} T^{-2}
$$

Изменение энтропии [28] находится из соотношений 


$$
\Delta S_{j}(T)=\Delta S_{j}(298)+\int_{298}^{T} \varsigma^{-1} c_{j p}(\varsigma) \mathrm{d} \varsigma
$$

Суммарное изменение свободной энергии равно положительному вкладу продуктов за вычетом вклада реагентов для каждой реакции.

Реакция горения углерода задается простейшей кинетикой

$$
\mathrm{O}_{2}+\mathrm{N}_{2}+\mathrm{C} \underset{Q_{1}}{\stackrel{k_{1}}{\longrightarrow}} \mathrm{CO}_{2}+\mathrm{N}_{2}-\left(H_{1}\right)
$$

Рассматривается схема синтеза титаната бария

$$
\mathrm{BaCO}_{3}+\mathrm{TiO}_{2} \rightarrow \mathrm{CO}_{2}+\mathrm{BaTiO}_{3}-\left(H_{2}\right)
$$

преимущества которой обсуждаются в [31]. Изменения свободной энергии Гиббса для реакций (1) и (2) равны

$$
H_{1}=\Delta G_{2}(T)-\Delta G_{1}(T)-\Delta G_{3}(T) \quad \text { и } \quad H_{2}=\Delta G_{2}(T)+\Delta G_{6}(T)-\Delta G_{4}(T)-\Delta G_{5}(T)
$$

Коэффициенты $a_{j}, b_{j}, c_{j}$ приводятся в термодинамических таблицах [24 ].

\section{2 Коэффициенты расширения}

Используем для компонент тензора напряжений обобщенные соотношения ДюамеляНеймана (для изотропного линейно упругого тела соотношения Дюамеля-Неймана, представляют закон Гука)

$$
\sigma_{i j}=2 \mu_{S 1} \varepsilon_{i j}+\delta_{i j}\left(\mu_{S 2} \sum_{k} \varepsilon_{k k}-K \omega\right)
$$

и соотношение термоупругости между инвариантами

$$
\sum_{k} \sigma_{k k}=\left(2 \mu_{S 1}+3 \mu_{S 2}\right) \sum_{k} \varepsilon_{k k}-3 K \omega
$$

следующее из соотношений Дюамеля-Неймана. Принимаем упрощенное воздействие газа на границы пор, а именно, допускаем что сумма нормальных напряжений в твердой фазе уравновешивается давлением газа на границу пор, т.е. $\sum_{k} \sigma_{k k}=-3 p$ [30]. Величины $\omega_{g}, \omega_{S}$ оцениваются через молярные объемы, занимаемые компонентами

$$
v_{\mathrm{lg}}=\frac{M_{\mathrm{lg}}}{\rho_{\mathrm{lg}}}, v_{j S}=\frac{M_{j S}}{\rho_{j S}} ; \quad \bar{\alpha}_{i S}=\frac{1}{3} \frac{v_{i S}}{\sum_{l=1}^{2} v_{\mathrm{lg}}+\sum_{j=1}^{4} v_{j S}}, i=1, \ldots, 4 ; \quad \bar{\alpha}_{i l}=\frac{1}{3} \frac{v_{l g}}{\sum_{l=1}^{2} v_{\mathrm{lg}}+\sum_{j=1}^{4} v_{j S}}, l=1,2,3
$$

- молярные концентрации компонентов с использованием соотношений

$$
\omega_{g}=1+3\left[\sum_{j=1}^{2} \alpha_{j g}\left(-B_{j g}+B_{j g}^{0}\right)\right] \quad \text { и } \quad \omega_{S}=3\left[\sum_{j=1}^{4} \alpha_{j S}\left(-B_{j S}+B_{j S}^{0}\right)\right],
$$

в которых

$$
\begin{gathered}
\alpha_{i g}=B_{i g} \bar{\alpha}_{i g}, \alpha_{i S}=B_{i S} \bar{\alpha}_{i S}, B_{j g}=\frac{Y_{j g}}{Y_{g, \text { tot }}}, B_{j S}=\frac{Y_{j S}}{Y_{S, \text { tot }}} Y_{\mathrm{lg}}=\frac{\rho_{\mathrm{lg}}}{M_{\mathrm{lg}}}, \quad Y_{g, \text { tot }}=\sum_{l=1}^{3} Y_{\mathrm{lg}}, \\
Y_{j S}=\frac{\rho_{j S}}{M_{j S}}, Y_{S, t o t}=\sum_{j=1}^{4} Y_{j S},
\end{gathered}
$$


индекс ноль относится к величинам в начальный момент времени [11].

Положим $\left(2 \mu_{S 1}+3 \mu_{S 2}\right)=3 \mu_{S}$, тогда

$$
\sum_{k} \varepsilon_{k k}=(K \omega-p) \mu_{S}^{-1}, \omega=\omega_{T}+\omega_{S}
$$

Далее эффект давления газа на поверхность пор не рассматривается $\sigma_{i i}=0$ и следовательно

$$
\sum_{k} \varepsilon_{k k}=K \omega \mu_{S}^{-1}
$$

Формула вычисления пористости

$$
\begin{gathered}
\chi(t, x, r)=\frac{V_{n}(t, x, r)}{V_{\Sigma}(t, x, r)}, \text { где } V_{\Sigma}(t, x, r)=V_{g}(t, x, r)+V_{S}(t, x, r) \\
\chi(t, x, r)=\frac{\chi_{0} \omega_{g}(t, x, r)}{\chi_{0} \omega_{g}(t, x, r)+\left(1-\chi_{0}\right)[1+E(t, x, r)]}, \\
E(t, x, r)=\sum_{k} \varepsilon_{k k}(t, x, r), \omega_{g}=1+3\left[\sum_{j=1}^{2} \alpha_{j g}\left(-B_{j g}+B_{j g}^{0}\right)\right],
\end{gathered}
$$

где

индекс ноль относится к величинам в начальный момент времени. Заметим, что если отсутствует эффект концентрационного расширения в газовой фазе, т.е. $\alpha_{j g}=0$, тогда

$$
\chi(t, x, r)=\frac{\chi_{0}}{\chi_{0}+\left(1-\chi_{0}\right)[1+E(t, x, r)]}
$$

Переходим к безразмерным переменным, отмеченные тильдой

$$
\begin{aligned}
& \tilde{x}_{i}=\frac{x_{i}}{l_{0}}, \tilde{t}=\frac{t}{t_{0}}, \tilde{u}_{i}=\frac{u_{i}}{u_{0}}, \tilde{V}_{i, \text { solid }}=\frac{V_{i, \text { solid }}}{u_{0}}, \tilde{p}=\frac{p}{p_{0}}, u_{0}=\frac{l_{0}}{t_{0}}, \quad i=1,2,3 ; \\
& \rho_{1 g}=\rho_{\mathrm{O}_{2}}, \rho_{2 g}=\rho_{\mathrm{CO}_{2}}, \rho_{3 g}=\rho_{\mathrm{N}_{2}}, \quad \rho_{g}=\rho_{1 g}+\rho_{2 g}+\rho_{3 g}, \quad \tilde{\rho}_{g}=\frac{\rho_{g}}{\rho_{0}}, \tilde{\rho}_{j g}=\frac{\rho_{j g}}{\rho_{0}}, \quad j=1,2,3 \text {; } \\
& \tilde{c}_{p g}=\frac{C_{p g}}{c_{p}}, \tilde{c}_{p g}=\frac{C_{p g}}{c_{p}}, \tilde{D}=\frac{D}{D_{0}}, \rho_{1 S}=\rho_{C}, \rho_{2 S}=\rho_{\mathrm{BaCO}_{3}}, \rho_{3 S}=\rho_{\mathrm{TiO}_{2}}, \rho_{4 S}=\rho_{\mathrm{BaTiO}_{3}}, \\
& \tilde{\rho}_{l S}=\frac{\rho_{l S}}{\rho_{0 C}}, \rho_{S}=\sum_{l=1}^{4} \rho_{l S}, \quad l=1, \ldots, 4 \\
& \tilde{c}_{p g}=\frac{C_{p g}}{c_{p}}, \zeta_{P}=\frac{\rho_{0}}{\rho_{0 C}}, \tilde{\kappa}_{0}=\frac{\kappa_{0} t_{0} A}{c_{p} \rho_{0} V}, \tilde{D}_{m}=\frac{D_{m}}{D_{0}}, \tilde{\lambda}_{g}=\frac{\lambda_{\text {air }}}{\lambda_{0}}, \tilde{\lambda}_{S}=\frac{\lambda_{S}}{\lambda_{0}}, \tilde{c}_{S}=\frac{C_{S}}{c_{p}}, \mathrm{Ma}^{-2}=\frac{\gamma_{\mathrm{air}} p_{0}}{\rho_{0} u_{0}^{2}} \\
& \mathrm{Re}=\frac{l_{0}^{2}}{t_{0} v_{\text {air }}}, \quad \mathrm{R}_{\text {solid }}=p_{0}\left(\zeta_{p} \mu_{S 0}\right)^{-1}, \quad \mathrm{Pe}_{T}=\frac{l_{0}^{2} \rho_{0} c_{p}}{t_{0} \lambda_{0}}, \quad \mathrm{Pe}_{1}=\frac{l_{0}^{2}}{t_{0} D_{0}}, \\
& \tilde{Q}=\frac{Q t_{0} k}{\rho_{0} C_{p g} T_{0}}, \tilde{k}=\frac{t_{0} k}{\rho_{0}}, \quad p_{0}=\frac{R \rho_{0} T_{0}}{M_{0}}, \quad \mu_{0}=\rho_{0} v_{\text {air }}, \quad \tilde{K}=\frac{K}{\mu_{S 0}}, \quad \tilde{\mu}_{\text {solid }}=\frac{\mu_{\text {solid }}}{\mu_{S 0}}
\end{aligned}
$$


Здесь $\quad l_{0}=0.007 \mathrm{~m}, \quad t_{0}=2.215(s), \quad u_{0}=3.25 \times 10^{-3} \mathrm{~m} / \mathrm{s}, \quad A=0.015 \mathrm{~m}^{2}, \quad V=1.1 \times 10^{-4} \mathrm{~m}^{3}$, $\rho_{0}=\rho_{\text {air }}=0.4 \mathrm{~kg} \times \mathrm{m}^{-3}, \lambda_{0}=\lambda_{\text {air }}=0.06(\mathrm{~W} / \mathrm{m} / \mathrm{K}), \quad D_{0}=2 \times 10^{-5} \mathrm{~m}^{2} / \mathrm{s}, \quad \mu_{S 0}=2 \times 10^{9} \mathrm{~Pa}$, $c_{p}=C_{p, \text { air }}=1114(\mathrm{~J} / \mathrm{kg} / \mathrm{K}), v_{\text {air }}=9.7 \times 10^{-5} \mathrm{~m}^{2} / \mathrm{s}, \rho_{0 C}=2267 \mathrm{~kg} \times \mathrm{m}^{-3}$, плотности компонент газовой и твердой фазы $\rho_{1 g}=\rho_{\mathrm{O}_{2}}, \rho_{2 g}=\rho_{\mathrm{CO}_{2}}, \rho_{3 g}=\rho_{\mathrm{N} 2}, \rho_{1 S}=\rho_{\mathrm{C}}, \rho_{2 S}=\rho_{\mathrm{BaCO}_{3}}, \rho_{3 S}=\rho_{\mathrm{BaO}}$, $\rho_{4 S}=\rho_{\mathrm{TiO}_{2}}, \rho_{5 S}=\rho_{\mathrm{BaTiO}_{3}}, \rho_{6 S}=\rho_{\mathrm{Ba}_{2} \mathrm{TiO}_{4}}$. Температура газовой смеси и смеси твердых компонент $T_{g}, T_{S}$. Температура находится по формуле $T_{g}=T_{0}\left(1+\beta \tilde{T}_{g}\right), T_{S}=T_{0}\left(1+\beta \tilde{T}_{S}\right)$, $\beta=R T_{0} / E$ - безразмерный параметр, характеризующий энергию активации; $R, E, Q$ газовая постоянная, энергия активации, тепловой эффект горения; давление газа $p=\rho_{g} T_{0}\left(1+\beta \tilde{T}_{g}\right)$; $\kappa_{0}$ - безразмерный коэффициент теплообмена; $\tilde{c}_{S}, \tilde{c}_{p g}$ - теплоемкости; $\tilde{\lambda}_{S}, \tilde{\lambda}_{g}-$ коэффициенты теплопроводности; $\tilde{D}$ - коэффициент диффузии, Мa, Re - числа Маха, Рейнольдса; $\mathrm{Pe}_{T}, \mathrm{Pe}_{1}$ - тепловое и диффузионное число Пекле; индекс “air” относится к параметрам для воздуха при нормальных условиях.

Приведем базовые уравнения форме, допускающей регулярный предельный переход $\chi \rightarrow 0$ и $\chi \rightarrow 1$. Система уравнений приводится ниже в безразмерных переменных, символ тильда опускаются.

Уравнение сохранения плотности фаз

$$
\begin{gathered}
\rho_{g}=\rho_{1 g}+\rho_{2 g}+\rho_{3 g}, \quad \rho_{S}=\sum_{j=1}^{4} \rho_{j S} \\
\frac{\partial \chi \rho_{g}}{\partial t}+\nabla \cdot\left(\chi \rho_{g} \mathbf{u}\right)=J_{S \rightarrow g}, \frac{\partial(1-\chi) \rho_{S}}{\partial t}=-J_{S \rightarrow g}, \\
J_{S \rightarrow g}=-\frac{M_{1 g}}{M_{1 S}} J_{1 S}+\frac{M_{2 g}}{M_{1 S}}\left(J_{1 S}+J_{2 S}\right)
\end{gathered}
$$

Уравнения сохранения массы компонент $\mathrm{O}_{2}, \mathrm{CO}_{2}, \mathrm{~N}_{2}$

$$
\begin{gathered}
\rho_{\mathrm{O}_{2}}=\rho_{g} C_{1}, \quad \rho_{\mathrm{CO}_{2}}=\rho_{g} C_{2}, \quad \rho_{\mathrm{N}_{2}}=\rho_{g} C_{3}, \\
\frac{\partial \chi \rho_{g} C_{1}}{\partial t}+\nabla \cdot\left(\chi \rho_{g} C_{1} \mathbf{u}\right)=\nabla \cdot\left(\frac{\chi}{P e_{1}} \rho_{g} \mathrm{D}_{\mathrm{mg}} \nabla C_{1}\right)-\frac{M_{1 g}}{M_{1 S}} J_{1 S}, \\
\frac{\partial \chi \rho_{g} C_{2}}{\partial t}+\nabla \cdot\left(\chi \rho_{g} C_{2} \mathbf{u}\right)=\nabla \cdot\left(\frac{\chi}{P e_{1}} \rho_{g} \mathrm{D}_{\mathrm{mg}} \nabla C_{2}\right)+\frac{M_{2 g}}{M_{1 S}}\left(J_{1 S}+J_{2 S}\right), C_{3}=1-C_{1}-C_{2}
\end{gathered}
$$

Компоненты тензора массовой дисперсии имеют вид $[7,10] \mathrm{D}_{\mathrm{mg}}=\left(\begin{array}{cc}D_{1 m} & 0 \\ 0 & D_{2 m}\end{array}\right)$

$$
\begin{gathered}
D_{1 m}=D_{m}\left[\xi+\varphi_{1}\left(P e_{1 m}\right)\right], \quad D_{2 m}=D_{m}\left[\xi+\varphi_{2}\left(P e_{2 m}\right)\right], \quad \mathrm{Pe}_{1 m}=\frac{|u| \cdot d_{p}}{D_{m}}, \mathrm{Pe}_{2 m}=\frac{|v| \cdot d_{p}}{D_{m}} \\
\varphi_{1}\left(\mathrm{Pe}_{1 m}\right)=\left(b_{0} \mathrm{Pe}_{1 m}+b_{1} \mathrm{Pe}_{1 m} \ln \mathrm{Pe}_{1 m}\right), \quad \varphi_{2}\left(\mathrm{Pe}_{2 m}\right)=\left(b_{0} \mathrm{Pe}_{2 m}+b_{1} \mathrm{Pe}_{2 m} \ln \mathrm{Pe}_{2 m}\right)
\end{gathered}
$$

Здесь $b_{0}, b_{1}$ - постоянные; $\xi$ - коэффициент извилистости пор ( $\left.\xi=0.5\right)$ [29].

В реакциях для компонент твердой фазы реагенты не смешиваются на молекулярном уровне, диффундируют и движутся в пределах твердой фазы могут быть записаны в форме 


$$
\begin{gathered}
\rho_{1 S}=\rho_{\mathrm{C}}, \quad \rho_{2 S}=\rho_{\mathrm{BaCO}_{3}}, \quad \rho_{3 S}=\rho_{\mathrm{TiO}_{2}}, \quad \rho_{4 S}=\rho_{\mathrm{BaTiO}_{3}}, \\
\frac{\partial(1-\chi) \rho_{1 S}}{\partial t}=-J_{1 S}, \quad \frac{\partial(1-\chi) \rho_{2 S}}{\partial t}=-\frac{M_{2 S}}{M_{1 S}} J_{2 S}, \\
\frac{\partial(1-\chi) \rho_{3 S}}{\partial t}=-\frac{M_{3 S}}{M_{1 S}} J_{2 S}, \frac{\partial(1-\chi) \rho_{4 S}}{\partial t}=\frac{M_{4 S}}{M_{1 S}} J_{2 S}
\end{gathered}
$$

Массовые потоки для реакций (1) и (2) равны соответственно:

$$
J_{1 S}=\chi(1-\chi) \rho_{1 S} \rho_{1 g} k_{1} \exp \left(\frac{T_{g}}{\beta T_{g}+1}\right), \quad J_{2 S}=(1-\chi)^{2} \rho_{2 S} \rho_{3 S} k_{2} \exp \left(\frac{T_{g}}{\beta T_{g}+1}\right)
$$

Уравнение движения газа в порах [2-10]

$$
\frac{\partial \chi \rho_{g} \mathbf{u}}{\partial t}+\nabla \cdot\left(\chi \rho_{g} \mathbf{u u}\right)+\mathrm{Ma}^{-2} \nabla p=\operatorname{Re}^{-1} \nabla \cdot \boldsymbol{\tau}+S_{V}, \boldsymbol{\tau}=\mu\left[\nabla \mathbf{u}+(\nabla \mathbf{u})^{T}-\frac{2}{3}(\nabla \cdot \mathbf{u}) \mathbf{I}\right],
$$

где слагаемое распределенного сопротивления газа в порах $\mathbf{S}_{\mathbf{V}}$ имеет вид

$$
\left(\mathbf{S}_{\mathbf{V}}\right)_{j}=-u_{j} \eta_{j}, \quad \eta_{j}=\eta_{1 j}|\mathrm{u}|+\eta_{0 j},
$$

где $u_{j}$ - компоненты скорости в Декартовой системе координат; $p=\rho_{g}\left(1+\beta T_{g}\right)-$ давление газовой фазы.

Уравнения движения твердой фазы запишем в простейшем случае, пренебрегая влиянием работы механических напряжений на поле температуры [19, 30].

$$
\frac{\partial(1-\chi) \boldsymbol{\rho}_{S} \mathbf{v}_{\text {solid }}}{\partial t}+\nabla \cdot\left[(1-\chi) \boldsymbol{\rho}_{S} \mathbf{v}_{\text {solid }} \mathbf{v}_{\text {solid }}\right]=\mathrm{R}_{\text {solid }}^{-1} \nabla \cdot \boldsymbol{\sigma}, \sigma_{i j}=2 \mu_{S 1} \varepsilon_{i j}+\delta_{i j}\left(\mu_{S 2} \sum_{k} \varepsilon_{k k}-K \omega\right),
$$

где $\mathrm{R}_{\text {solid }}$ - число Рейнольдса твердой фазы.

Уравнение баланса тепла в газовой фазе, включающее дисперсию, записывается в виде

$$
\rho_{g} c_{p g} \chi\left(\frac{\partial T_{g}}{\partial t}+\mathbf{u} \cdot \nabla T_{g}\right)+c_{g} T_{g} \chi J_{S \rightarrow g}=\nabla \cdot\left(\chi \frac{D_{T g}}{\mathrm{Pe}_{T g}} \nabla T_{g}\right)-\kappa \chi(1-\chi)\left(T_{g}-T_{S}\right)+\chi Q_{r}
$$

В правую часть уравнения включен тепловой поток $Q_{r}$ химического превращения $Q_{r}=-H_{1}-H_{2}$. Тензор тепловой дисперсии $[14,15]$ учитывает продольную и поперечную дисперсию

$$
D_{T g}=\left(\begin{array}{cc}
\lambda_{1 g} & 0 \\
0 & \lambda_{2 g}
\end{array}\right), \lambda_{1 g}=\lambda_{g}\left[\xi+\varphi_{1}\left(\mathrm{Pe}_{1 t}\right)\right], \lambda_{2 g}=\lambda_{g}\left[\xi+\varphi_{2}\left(\mathrm{Pe}_{2 t}\right)\right]
$$

Уравнение баланса тепла в твердой фазе имеет вид

$$
\begin{aligned}
\rho_{S} c_{S}(1-\chi)\left(\frac{\partial T_{S}}{\partial t}+\mathrm{V}_{\text {solid }} \cdot \nabla T_{S}\right)-c_{S} T_{S}(1-\chi) J_{S \rightarrow g}= \\
=\nabla \cdot\left[(1-\chi) \frac{\lambda_{S}}{P e_{T S}} \nabla T_{S}\right]+\kappa \chi(1-\chi)\left(T_{g}-T_{S}\right)+(1-\chi) Q_{r}
\end{aligned}
$$

Коэффициент теплообмена между газовой и твердой фазами описывается с помощью формулы Левека [3, 33] в виде 


$$
\kappa=\kappa_{0}\left(1+\operatorname{Re}_{\text {loc }}^{0.3} \mathrm{Pe}_{\mathrm{Tloc}}^{0.3}\right)
$$

Здесь $\operatorname{Re}_{\text {loc }}=\operatorname{Re}|\mathbf{u}| \rho_{g}, \operatorname{Pe}_{\text {Tloc }}=\operatorname{Pe}_{T g}|\mathbf{u}| \rho_{g}, \operatorname{Pe}_{\text {Tloc }}=\operatorname{Pe}_{T}|\mathbf{u}| \rho_{g}$ - локальные числа Рейнольдса и Пекле [3], (скорость и плотность - безразмерные! $\left.\operatorname{Re}_{\text {loc }}=\operatorname{Re}|\tilde{\mathbf{u}}| \tilde{\rho}_{g}, \operatorname{Pe}_{\mathrm{Tloc}}=\operatorname{Pe}_{T g}|\tilde{\mathbf{u}}| \tilde{\rho}_{g}\right)$.

\section{3 Граничные и начальные условия для трехзонного реактора}

Граничные условия на входе в зоны

$$
\begin{aligned}
& t>0 ; x=0 ; 0<r<R_{2}: u=0, v=0, \frac{\partial T_{g}}{\partial n}=\alpha_{e x}\left(T_{e x}-T_{g}\right), \frac{\partial C_{i}}{\partial n}=0 ; \\
& t>0, x=0: u=u_{1}, p=p_{0}, T=T_{\text {init }}, t>0, x=L: \frac{\partial u}{\partial x}=0, \frac{\partial p}{\partial x}=0, \frac{\partial T}{\partial x}=0 ; \\
& t>0 ; x=0 ; R_{1}<r<1: u=u_{i n}, v=0, \frac{\partial \mathrm{T}_{g}}{\partial n}=\alpha_{e x}\left(T_{e x}-T_{g}\right), \frac{\partial C_{i}}{\partial n}=0 ; \\
& t>0 ; x=0 ; R_{2}<r<R_{1}: \frac{\partial \mathrm{T}_{g}}{\partial n}=-q_{f}(t), \frac{\partial \mathrm{T}_{S}}{\partial n}=-q_{f}(t), \frac{\partial C_{1}}{\partial n}=-J_{\mathrm{O}_{2}}, \frac{\partial C_{2}}{\partial n}=0, \frac{\partial C_{3}}{\partial n}=-J_{\mathrm{N}_{2}}, \\
& \frac{\partial \rho_{g} C_{1}}{\partial n}=-O_{2 f}, \frac{\partial \rho_{g} C_{3}}{\partial n}=-O_{2 f},, \\
& t>0 ; x=0 ; 0<r<R_{2}: u=0, v=0, \frac{\partial \mathrm{T}_{g}}{\partial n}=\alpha_{e x}\left(T_{e x}-T_{g}\right), \frac{\partial C_{i}}{\partial n}=0
\end{aligned}
$$

Граничные условия на выходе из зон

$$
\begin{aligned}
& t>0 ; x=L ; R_{1}<r<1: u=0, v=0, \frac{\partial \mathrm{T}_{g}}{\partial n}=\alpha_{e x}\left(T_{e x}-T_{g}\right), \frac{\partial C_{i}}{\partial n}=0 ; \\
& t>0 ; x=L ; R_{2}<r<R_{1}: \frac{\partial T_{g}}{\partial n}=\alpha_{e x}\left(T_{e x}-T_{g}\right), \frac{\partial T_{S}}{\partial n}=\alpha_{e x}\left(T_{e x}-T_{S}\right), \frac{\partial C_{i}}{\partial n}=0 ; \\
& t>0 ; x=L ; 0<r<R_{2}: \frac{\partial T_{g}}{\partial n}=\alpha_{e x}\left(T_{e x}-T_{g}\right), \frac{\partial C_{i}}{\partial n}=0
\end{aligned}
$$

Граничное условие на наружной стенке

$t>0 ; 0<x<L ; r=1: u=0, v=0, \frac{\partial \mathrm{T}_{g}}{\partial n}=\alpha_{e x}\left(T_{e x}-T_{g}\right), \frac{\partial C_{i}}{\partial n}=0, i=1,2,3$

Граничные условия на оси симметрии реактора

$t>0 ; 0<x<L ; r=0: \frac{\partial u}{\partial r}=0, v=0, \frac{\partial T_{g}}{\partial r}=0, \frac{\partial C_{i}}{\partial r}=0$

Условия на внутренней границы первой зоны $G_{1}=\left\{0<x<L ; R_{1}<r<1\right\}$

$t>0 ; 0<x<L ; r=R_{1}: T_{S}=T_{g}$ либо условие охлаждения $T_{S}=T_{g}=T_{W, 1}$

Условия на внутренней границы второй зоны $G_{2}=\left\{0<x<L ; R_{2}<r<R_{1}\right\}$ то же, что и выше

$t>0 ; 0<x<L ; r=R_{1}: T_{S}=T_{g}$, либо условие охлаждения $T_{S}=T_{g}=T_{W, 1} ;$

$t>0 ; 0<x<L ; r=R_{2}: T_{S}=T_{g}$, либо условие охлаждения $T_{S}=T_{g}=T_{W, 1}$

Условия на внутренней границы третьей зоны $G_{3}=\left\{0<x<L ; 0<r<R_{3}\right\}$ то же, что и выше

$t>0 ; 0<x<L ; r=R_{2}: T_{S}=T_{g}$, либо условие охлаждения $T_{S}=T_{g}=T_{W, 1}$

Начальные условия для каждой из трех зон

$t=0 ; 0<x<L ; R_{1}<r<1: u=u_{\text {in }}, v=0, T_{g}=T^{0}, C_{1}=C_{\mathrm{O}_{2}}^{0}, C_{2}=0, C_{3}=C_{\mathrm{N}_{2}}^{0}$ 


$$
\begin{aligned}
& t=0 ; 0<x<L ; R_{2}<r<R_{1}: u=0, v=0, T_{g}=T_{\text {init }}(x), T_{S}=T_{\text {init }}(x) ; \\
& C_{1}=\chi C_{\mathrm{O}_{2}}^{0}, C_{2}=0, C_{3}=\chi C_{\mathrm{N}_{2}}^{0}, \rho_{j S}=\rho_{j S}^{0}, j=1, \ldots, 6 ; \\
& t=0 ; 0<x<L ; 0<r<R_{2}: u=0, v=0, T_{g}=T^{0}, C_{1}=C_{\mathrm{O}_{2}}^{0}, C_{2}=0, C_{3}=C_{\mathrm{N}_{2}}^{0} ; \\
& q_{f}(t)=Q_{f}, 0 \leq t \leq t_{\text {init }} ; q_{f}(t)=0, t \geq t_{\text {init }}
\end{aligned}
$$

Предполагается свободный теплообмен на боковой границе $r=1$ и верхней границе $x=L$ при коэффициенте теплообмена $\alpha_{\mathrm{ex}}=10^{3}$.

\section{3. Результаты моделирования и обсуждение}

Представленные результаты моделирования синтеза титаната бария в реакторе с порами микронного размера проведены при следующих безразмерных параметрах ([7]): $t_{\text {init }}=0.25 \div 0.5, \beta=0.075 \div 0.1, \gamma \approx 0.288, \chi=0.2$ и $0.6, \alpha_{j}=0, \varsigma_{j}=50, K=56.4, \mathrm{Ma}=0.2$, $Q_{f}=10 \div 500, Q=60, \mathrm{Re}=0.24, \mathrm{Pe}_{T g}=0.2, \mathrm{Pe}_{T s}=0.6, \zeta_{p}=3.6 \times 10^{-4}, \mathrm{R}_{\text {solid }}=0.0316$.

Инициация горения производится тепловым потоком $q_{f}(t), q_{f}(t)=Q_{f}, 0 \leq t \leq t_{\text {init }}$, $q_{f}(t)=0, t \geq t_{\text {init }}$.

Задавался поток кислорода $\mathrm{O}_{2 f}(t)=1 \div 100$ на интервале времени $0<t<t_{2}$ поступающий с сечения $x=0, R_{2}<r<1$ рис. $1, \mathrm{~A}$. В начальный момент времени задавались плотности компонент твердой и газовой фазы. Скорость газа равна нулю в начальный момент времени и на внешней границе $0 \leq x \leq L, r=1$.

Расчеты тепловой дисперсии проведены при начальной пористости $\chi=0.6$ и диаметре частиц $d_{p}=3 \times 10^{-4}$ м. Для инициации горения задавался поток тепла на входе в реактор $Q_{f}=500, t_{\text {init }}=0.5$. В начальный момент времени заданы следующие коэффициенты расширения:

$$
\begin{gathered}
t=0: \bar{\alpha}_{l S}=\alpha_{l S}^{0}, l=1, \ldots, 4, \alpha_{1 S}^{0}=0.018, \alpha_{2 S}^{0}=0.653, \alpha_{3 S}^{0}=0.295, \alpha_{4 S}^{0}=2 \times 10^{-3}, \\
\alpha_{1 g}^{0}=4 \times 10^{-4}, \alpha_{2 g}^{0}=0.012, \alpha_{3 g}^{0}=7 \times 10^{-5},
\end{gathered}
$$

где $\alpha_{l s}^{0}$ - начальные коэффициенты концентрационного расширения. Расчеты проведены при нулевых начальных величинах концентрационного расширения газовых компонент. Коэффициент температурного расширения $\alpha_{T}=\alpha_{T}^{0}=5 \times 10^{-5}$ предполагался постоянным, На рис. 1-8 представлены результаты в случае $\alpha_{1 g}^{0}=0, \alpha_{2 g}^{0}=0, \alpha_{3 g}^{0}=0$. Влияние концентрационного расширения газовой фазы представлено на рис. 9-12 в случае (5), (6).

1. Данные расчетов для трехзонного осесимметричного реактора (рис. 1, А) в области моделирования $0<x<L, 0<r<1$, включающей канал подачи смеси $\mathrm{O}_{2}, \mathrm{~N}_{2}$ (зона 1): $0<x<L, 0<r<R_{1}$, пористую область смеси порошков углерода, реагентов и продуктов синтеза (зона 2): $0<x<L, R_{2}<r<R_{1}$ и канал отвода смеси $\mathrm{N}_{2}$ и $\mathrm{CO}_{2}$ (зона 3): $0<x<L, R_{1}<r<1$ представлены на рис. $1, \mathrm{~B}$, рис. $1, \mathrm{~B} 1$ и рис. $1, \mathrm{C}$, рис. $1, \mathrm{C} 1$. Показаны распределение и шкала температуры твердой фазы в момент времени $t=0.88$ на рис. $1, \mathrm{~B}$, рис. $1, \mathrm{~B} 1$ и распределение пористости в момент времени $t=0.88$ на рис. $1, \mathrm{C}$ и рис. $1, \mathrm{C} 1$. Расчет проведенный при (4) указывает на существенную неравномерность распределения температуры поперек пористой зоны реактора. Наиболее прогретой оказывается область вблизи границы $r=R 2$. Можно отметить уменьшение пористости от начальной величины 0.6 до 0.3 в зоне прореагировавшей смеси реагентов твердой фазы, вызванное эффектом концентрационного расширения. 


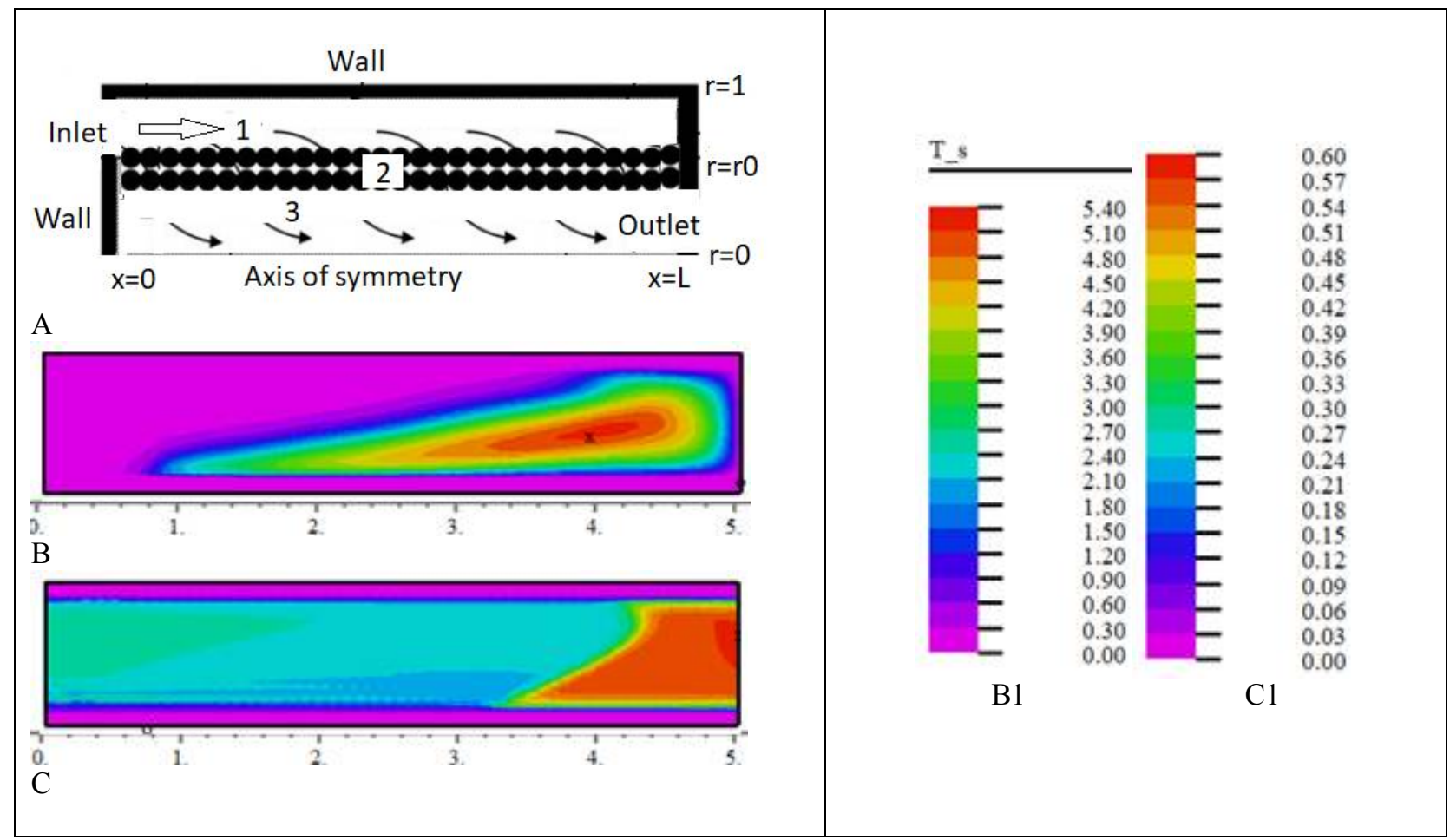

Рис. 1. Схема трехзонного осесимметричного реактора (А). Показана область моделирования $0<x<L, 0<r<1$.

Зона 1: $0<x<L, 0<r<R_{1}-$ канал отвода смеси $\mathrm{N}$, и $\mathrm{CO}$. . Зона 2: $0<x<L, R_{2}<r<R_{1}-$ пористая область смеси порошков углерода, реагентов и продуктов синтеза. Зона 3: $0<x<L, R_{1}<r<1$ канал подачи смеси $\mathrm{O}_{2}, \mathrm{~N}_{2}$ на рис. 1 , В и рис. $1, \mathrm{~B} 1$ показаны распределение и шкала температуры твердой фазы в момент времени $t=0.88$. На рис. $1, \mathrm{C}$ и рис. $1, \mathrm{C} 1$ представлено распределение пористости в момент времени $t=0.88$

2. Распределение продольной скорости твердой фазы. на рис. 2 , А, рис. 2 , А1 и рис. 2, В, рис. 2 , В1, представленное в моменты 0.2 и 0.88 соответственно позволяет наблюдать распространение волны продольного сжатия твердой фазы в процессе химических превращений согласно кинетике синтеза (1), (2).

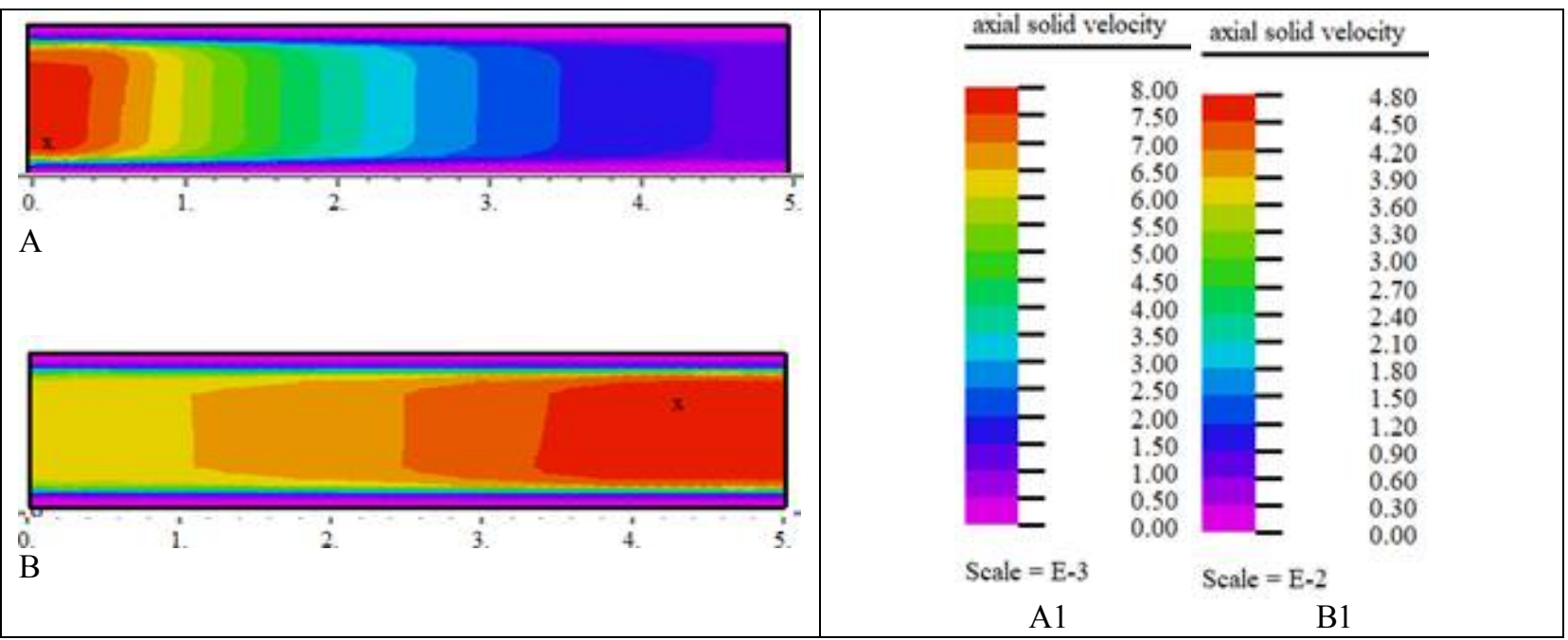

Рис. 2. Иллюстрируется динамика скорости твердой фазы. На рис. 2, А, рис. 2, А1 и рис. 2, В, рис. 2, В1 представлена продольная скорость твердой фазы в моменты $t=0.2, t=0.88$ соответственно 
Динамика пористости, показанная распределениями на рис. 3, А, рис. 3, В и рис. 3, С и шкалой на рис. $3, \mathrm{~A} 1$, рис. $3, \mathrm{~B} 1$ и рис. $3, \mathrm{C} 1$ в моменты $t=0.2,0.5$ и 0.88 соответственно иллюстрирует процесс уплотнения при синтезе титаната бария.

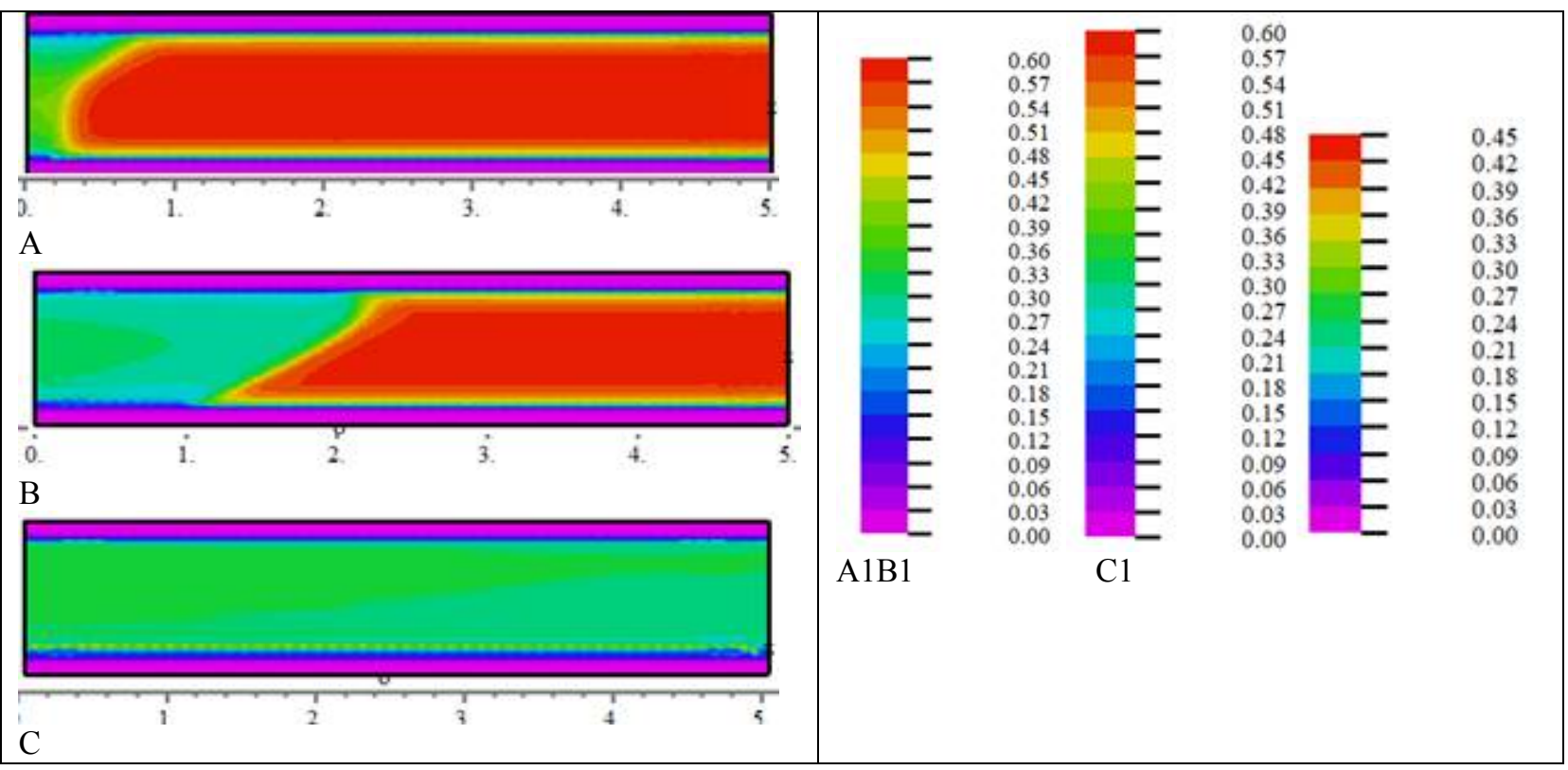

Рис. 3. Представлена динамика пористости. На рис. 3, А, рис. 3, В и рис. 3, С показана пористость в моменты $t=0.2,0.5$ и 0.88 , соответственно. На рис. 3 , A1, pис. 3 , В1 и рис. $3, \mathrm{C} 1$ приведена соответствующая шкала пористости

Сравнение динамики температуры газа $T_{g}\left(t, x, r_{0}\right)$ и твердой фазы $T_{S}\left(t, x, r_{0}\right)$ в контрольных точках $(x, r)$ с координатами $(0,0.5),(0.15,0.5),(0.375,0.5),(0.75,0.5),(1.125,0,5)$, $(1,5,0.5),(1.875,0.5),(2.25,0.5)$. Рисунок 4 , А и рис. 4 , В указывают на превалирующий рост температуры газа по мере движения фронта горения в пористой части реактора.

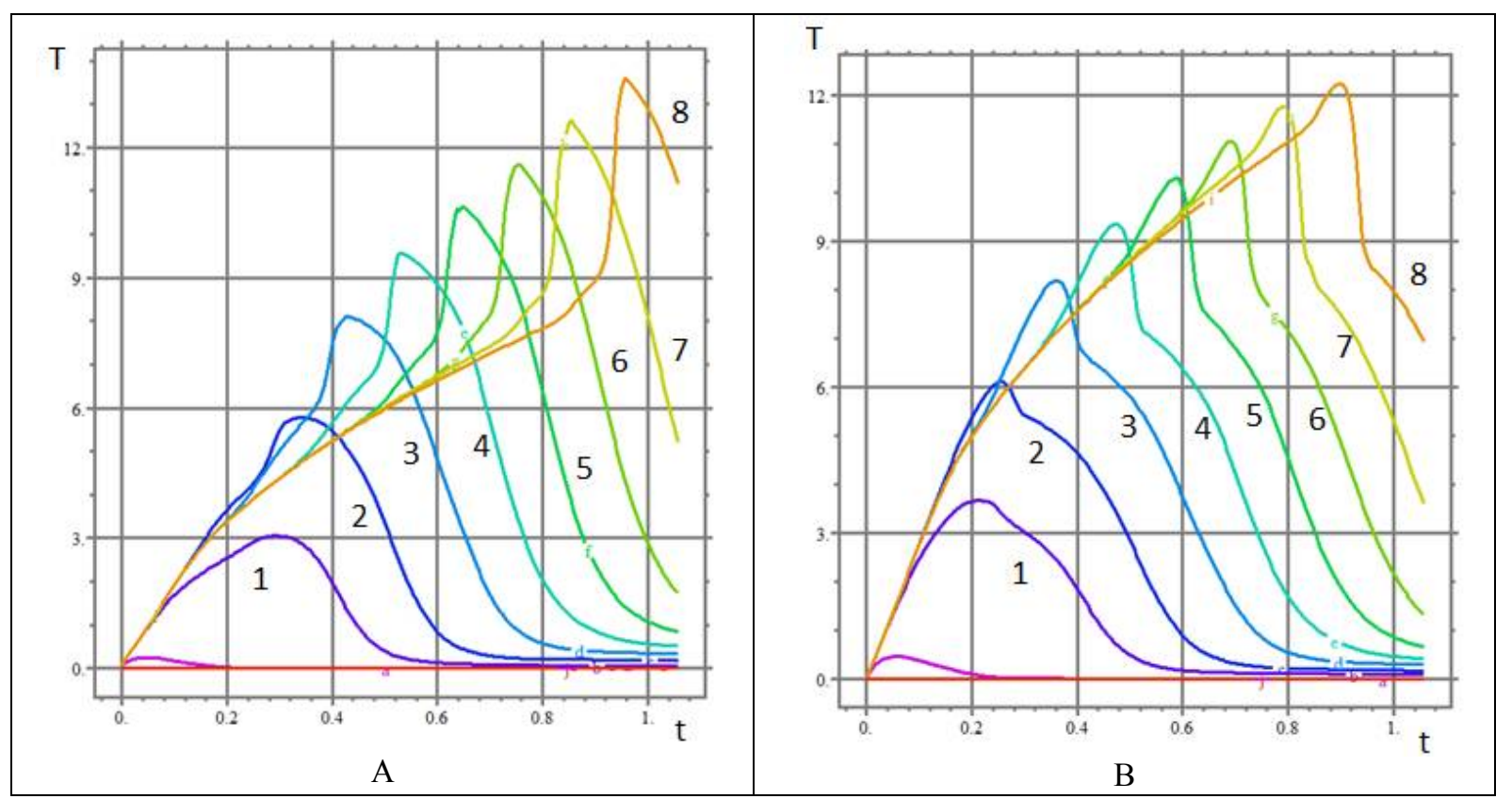

Рис.4. Иллюстрируется влияние коэффициентов расширения (5) на динамику температуры $T_{g}\left(t, x, r_{0}\right)$ газа и $T_{S}\left(t, x, r_{0}\right)$ твердой фазы А и В в контрольных точках $(x, r)$. Кривые $1-8$ относятся к точкам с координатами $(0,0.5),(0.15,0.5),(0.375,0.5),(0.75,0.5),(1.125,0.5),(1.5,0.5)$, $(1.875,0.5),(2.25,0.5)$ соответственно 
Сравнение температуры в центре пористой зоны реактора указывает на формирование во времени более прогретых областей при малом расширении вблизи входного сечения и в центральной части, однако в области пористой зоны, удаленной от входного сечения температура газа увеличивается с ростом объемного расширения. Заметим, что моделирование проводилось при большой величине коэффициента межфазового обмена. В наших расчетах при постоянной пористости $[7,10]$ температуры газа и смеси порошков реагентов и продукта синтеза отличались незначительно при том же межфазовом теплообмене. Результаты данной работы позволяют утверждать существенное влияние переменной пористости на межфазовый теплообмен.

На рис. 5 иллюстрируется волновой процесс тепловыделения первой и второй реакций (1), (2) (см. рис. 5, А и рис. 5, В, соответственно). Расчетные данные полученные при коэффициентах расширения (5) показывают экзотермический и эндотермический 1-й и 2-й реакции синтеза (1), (2). Результат на рис. 5 , А и рис. 5 , В показывает изменение во времени тепловыделения в контрольных точках $(x, r)$ с координатами $(0,0.5),(0.15,0.5),(0.375,0.5)$, $(0.75,0.5),(1.125,0,5),(1,5,0.5),(1.875,0.5),(2.25,0.5)$.
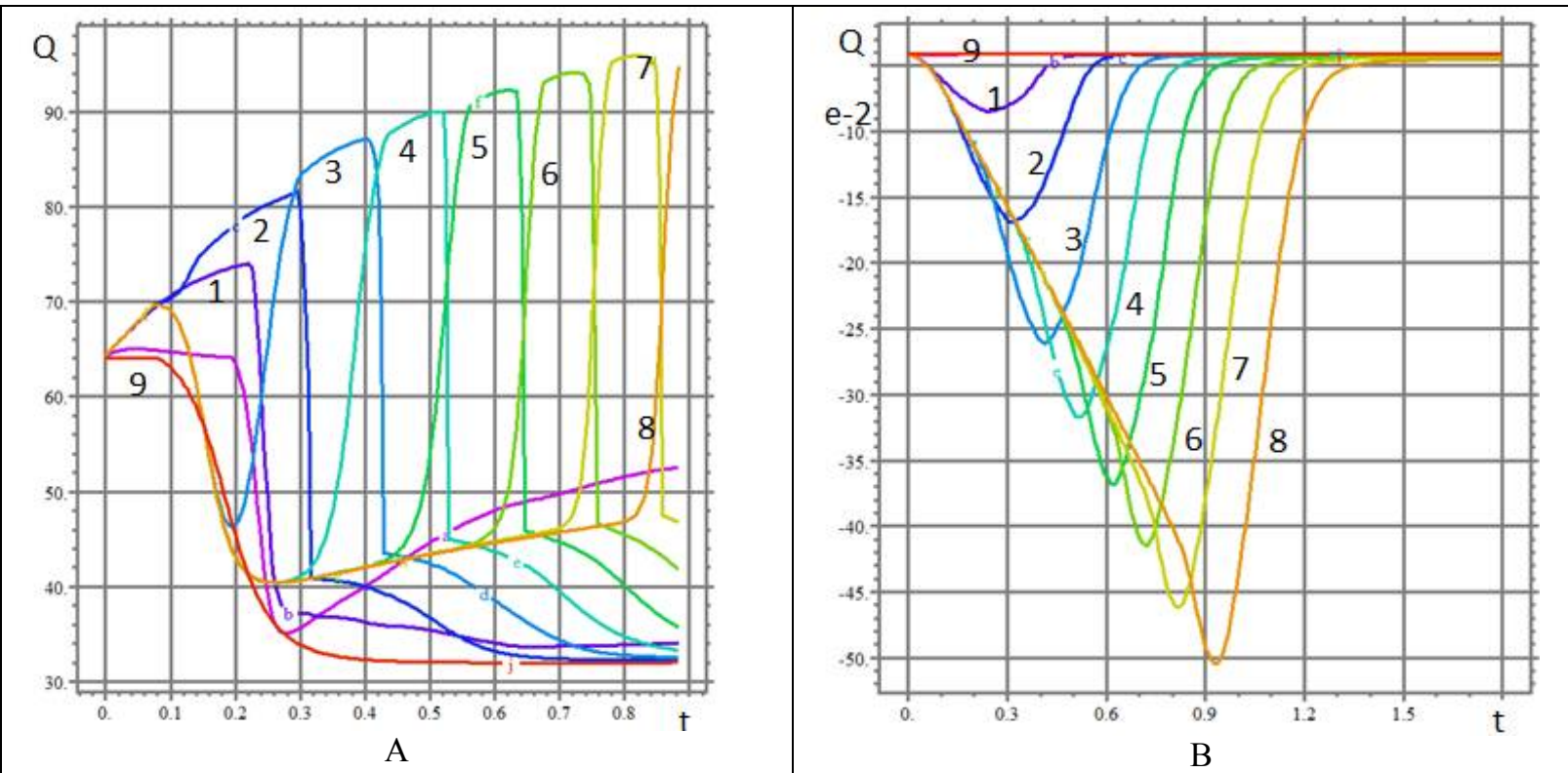

Рис. 5. Влияние коэффициентов расширения (5) на динамику тепловыделения 1-й и 2-й реакции синтеза (1), (2) иллюстрируется на А и В в контрольных точках с координатами $(0,0.5),(0.15,0.5)$, $(0.375,0.5),(0.75,0.5),(1.125,0.5),(1.5,0.5),(1.875,0.5),(2.25,0.5)$ (кривые 1-8 соответственно)

Динамика уплотнения твердой фазы в сечениях $r=\mathrm{R}_{1}$ и $r=r_{0}$ при коэффициентах концентрационного расширения (4) представлена на рис. 6 зависимостью от времени пористости в контрольных точках $(x, r)$ с координатами: $(0,0.25),(0.15,0.25),(0.75,0.25),(1.5,0.25)$, $(2.25,0.25),(3,0.25)$ - рис. 6 , А и $(0,0.5),(0.15,0.5),(0.75,0.5),(1.5,0.5),(2.25,0.5),(3,0.5)-$ рис. 6, В.

Расчетные данные на рис. 7 показывают динамику реагента $\mathrm{TiO}_{2}$ и продукта синтеза $\mathrm{BaTiO}_{3}$. В сечении $r=r_{0}$. Иллюстрируется влияние коэффициента концентрационного расширения (4) на зависимость от времени плотности $\mathrm{TiO}_{2}$, (рис. $7, \mathrm{~A}$ ) и $\mathrm{BaTiO}_{3}$ (рис. 7,В) в контрольных точках $(x, r)$ с координатами $(0,0.5),(0.15,0.5),(0.75,0.5),(1.5,0.5),(2.25,0.5)$, $(3,0.5)$. Уплотнение приводит к появлению зон локального максимума (рис. $7, B)$ истинной плотности продукта синтеза (массы отнесенной к объему твердой фазы).

Соответствующие результаты динамики реагентов $\mathrm{C}$ и $\mathrm{BaCO}_{3}$ в сечении $r=r_{0}$ представлены на рис. 8. Влияние коэффициента концентрационного расширения (5) на динамику плотности $\mathrm{C}$, (рис. $8, \mathrm{~A}$ ) и реагента $\mathrm{BaCO}_{3}$ (рис. $8, \mathrm{~B}$ ) иллюстрируется в контрольных точках $(x, r)$ с координатами $(0,0.5),(0.15,0.5),(0.75,0.5),(1.5,0.5),(2.25,0.5),(3,0.5)$. 


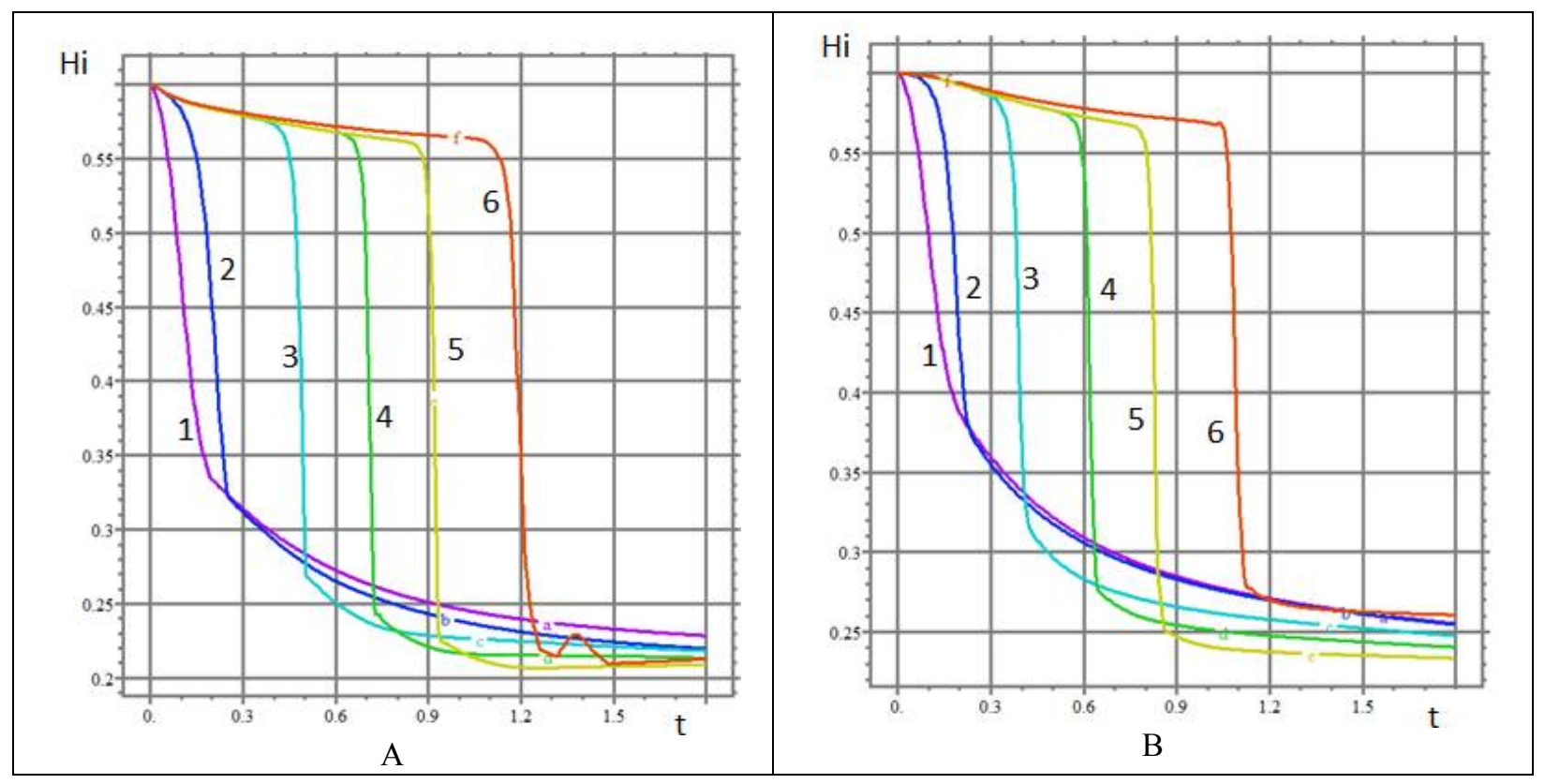

Рис. 6. Представлено влияние коэффициента концентрационного расширения (5) на динамику пористости в контрольных точках $(x, r)$ с координатами: $(0,0.25),(0.15,0.25),(0.75,0.25),(1.5,0.25)$, $(2.25,0.25),(3,0.25)$ (см. кривые $1-6$ на А) и в контрольных очках $(0,0.5),(0.15,0.5),(0.75,0.5)$, $(1.5,0.5),(2.25,0.5),(3,0.5)$ (см. кривые 1-6 на В)

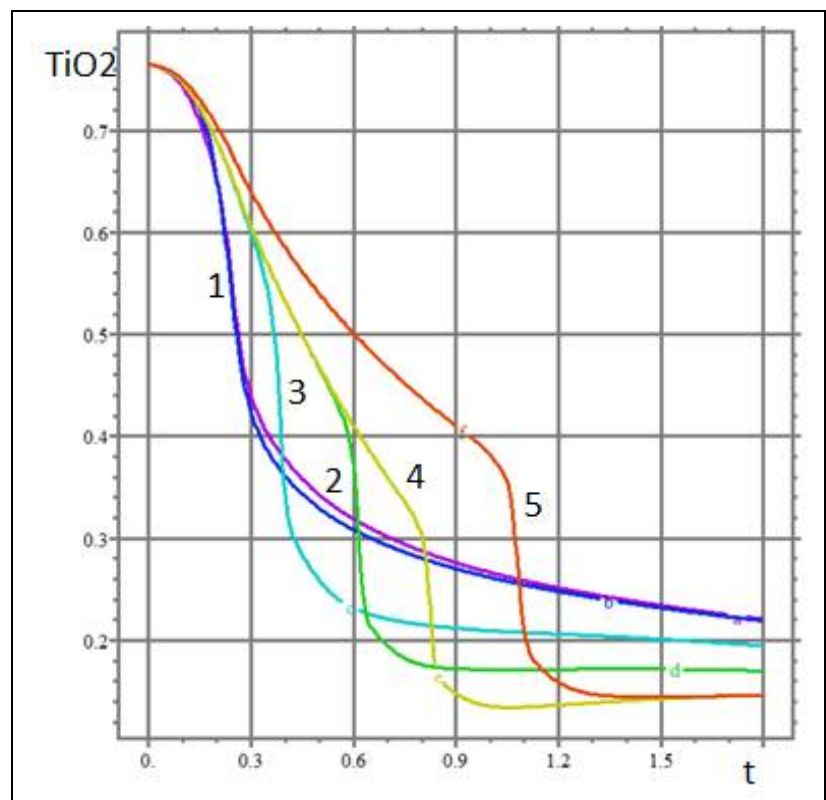

A

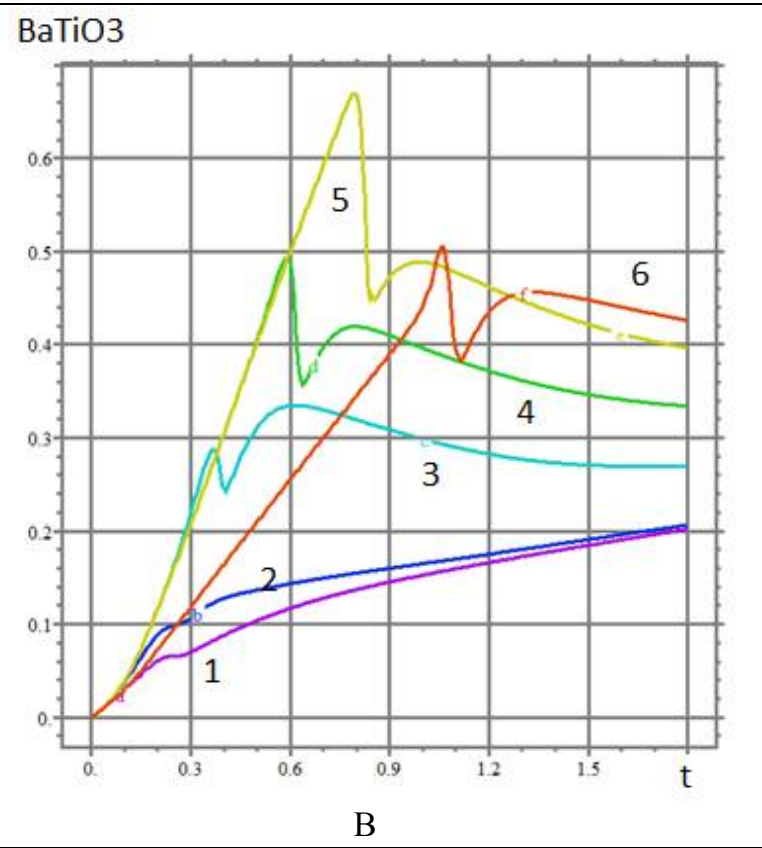

Рис. 7. Влияние коэффициента концентрационного расширения (5) на динамику плотности $\mathrm{TiO}_{2}$, (A) и $\mathrm{BaTiO}_{3}(\mathrm{~B})$. Кривые $1-8$ относятся к точкам с координатами $(0,0.5),(0.15,0.5),(0.75,0.5)$, $(1.5,0.5),(2.25,0.5),(3,0.5)$ 


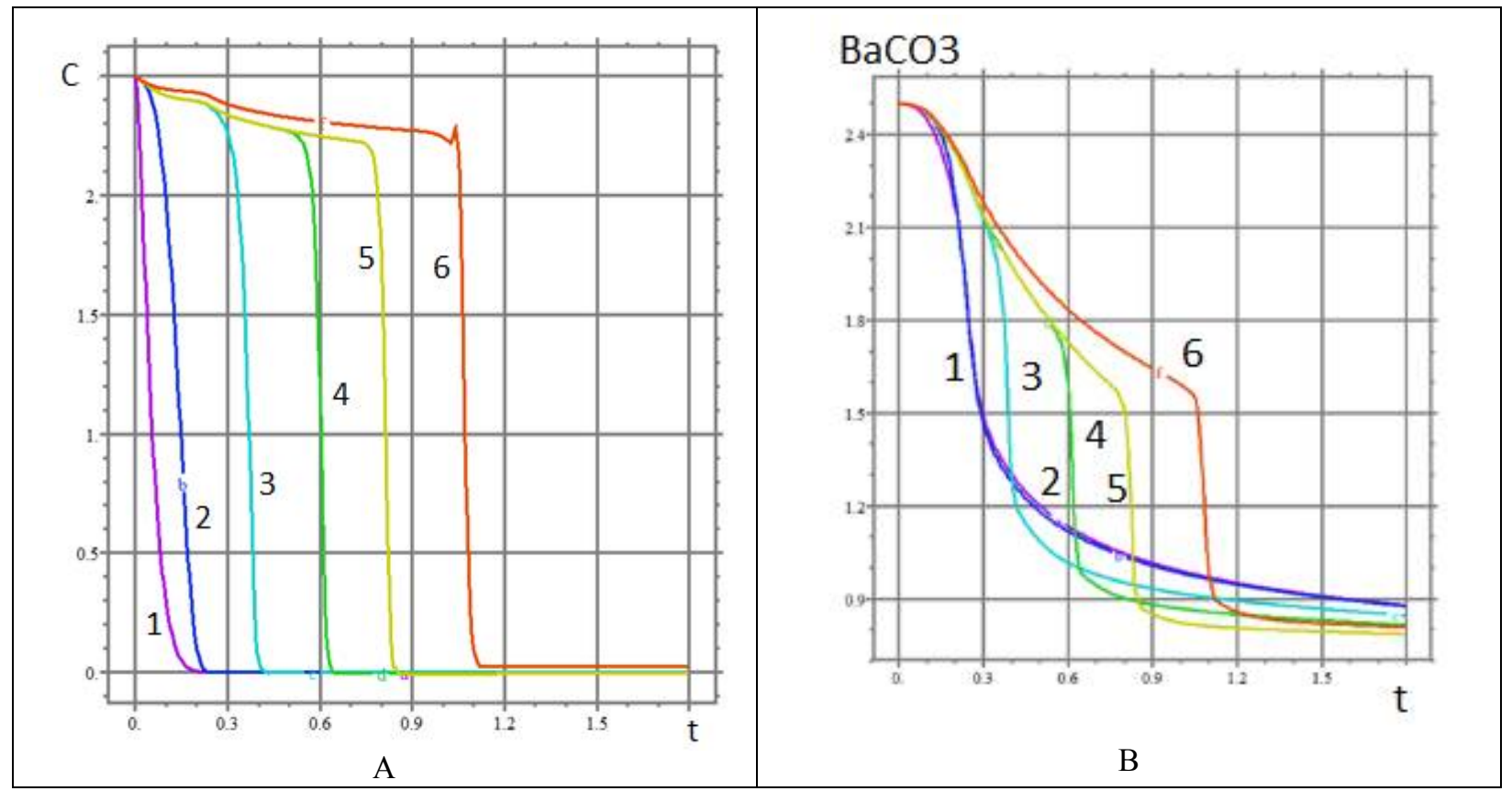

Рис. 8. Влияние коэффициента концентрационного расширения (5) на динамику плотности С (А) и $\mathrm{BaCO}_{3}(\mathrm{~B})$ в контрольных точках $(x, r)$ с координатами $(0,0.5),(0.15,0.5),(0.75,0.5),(1.5,0.5)$, $(2.25,0.5),(3,0.5)$ (кривые 1-6). Динамика $\mathrm{C}, \mathrm{BaCO}_{3}$ представлена в сечении $r=r_{0}$

На рис. 9-12 представлены результаты моделирования работы реактора при одновременном воздействии концентрационного расширения как газовой, так и твердой фазы.

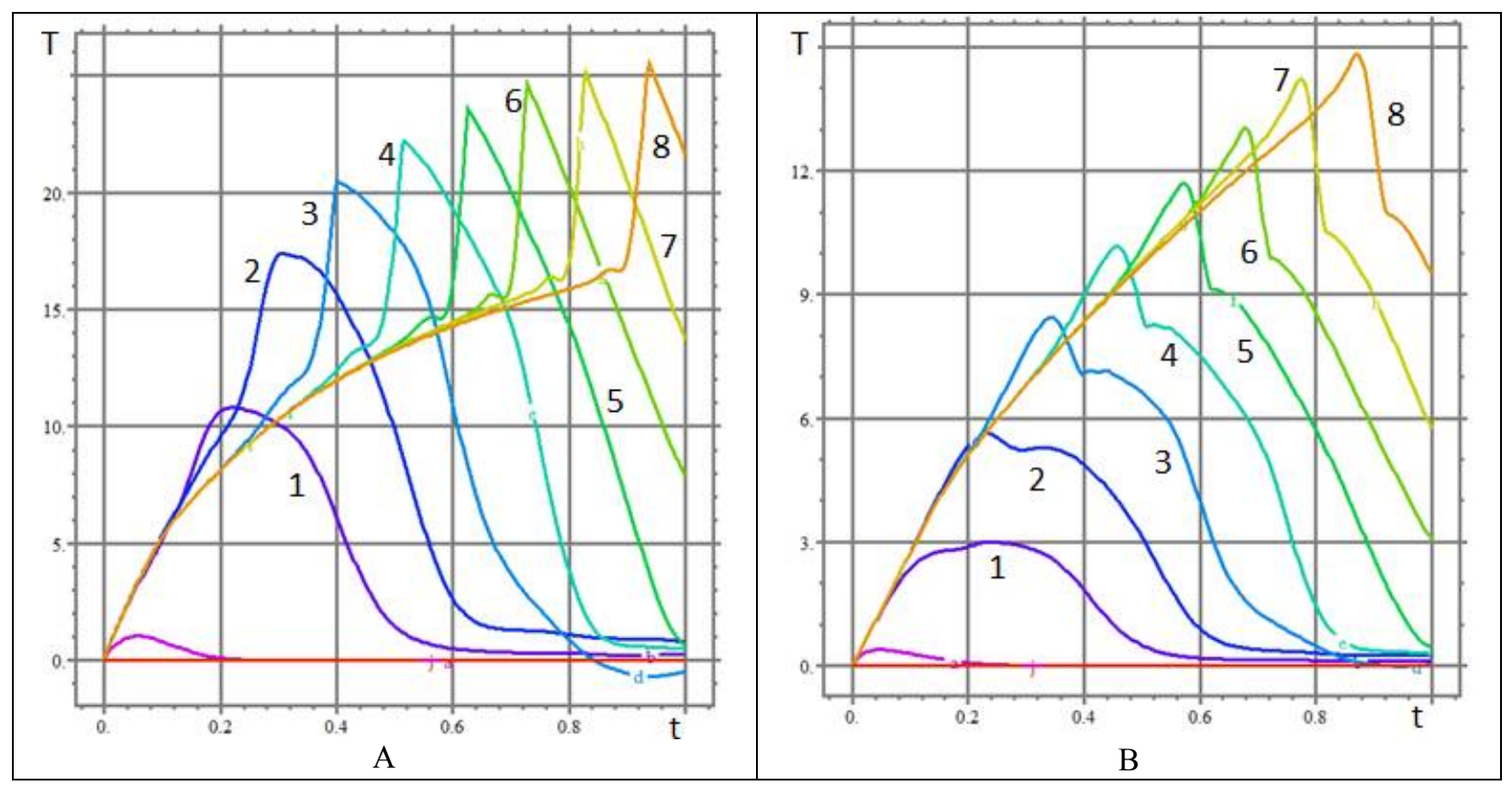

Рис. 9. Концентрационное расширение газовой и твердой фаз. Сравнение динамики температуры газа $T_{g}\left(t, x, r_{0}\right)$ при начальной пористости $\chi_{0}=0.2(\mathrm{~A})$ и $\chi_{0}=0.6(\mathrm{~B})$ в контрольных точках с координатами $(0,0.5),(0.15,0.5),(0.375,0.5),(0.75,0.5),(1.125,0.5),(1.5,0.5),(1.875,0.5),(2.25,0.5)$ показано кривыми 1-8 соответственно

При начальной пористости $\chi_{0}=0.6$ температура газа $T_{g}\left(t, x, r_{0}\right)$ примерно в два раза меньше, чем при $\chi_{0}=0.2$. Температура твердой фазы $T_{S}\left(t, x, r_{0}\right)$ достигает больших величин при $\chi_{0}=0.6$, т.к. согласно рис. 11 , удельный объем твердой фазы $1-\chi$ оказывается меньше 
$\chi_{0}=0.6$, чем при $\chi_{0}=0.2$. Сравним теперь воздействие концентрационного расширения газовой фазы для $\chi_{0}=0.6$ на примере динамики $T_{g}\left(t, x, r_{0}\right)$, полученной при нулевых начальных коэффициентах $\alpha_{1 g}^{0}=0, \alpha_{2 g}^{0}=0, \alpha_{3 g}^{0}=0$ на рис. 4 , А и ненулевых коэффициентах (6) на рис. 9 , В. Можно видеть более сильный прогрев газа на рис. 9 динамика и значительное отличие динамики прогрева. Аналогичное заключение о превалирующем нагревании твердой фазы $T_{S}\left(t, x, r_{0}\right)$ на рис. $10, \mathrm{~B}$ и различии динамики температуры $T_{S}\left(t, x, r_{0}\right)$ твердой фазы можно получить сравнением данных на рис. 4 , В и рис. $10, \mathrm{~B}$.

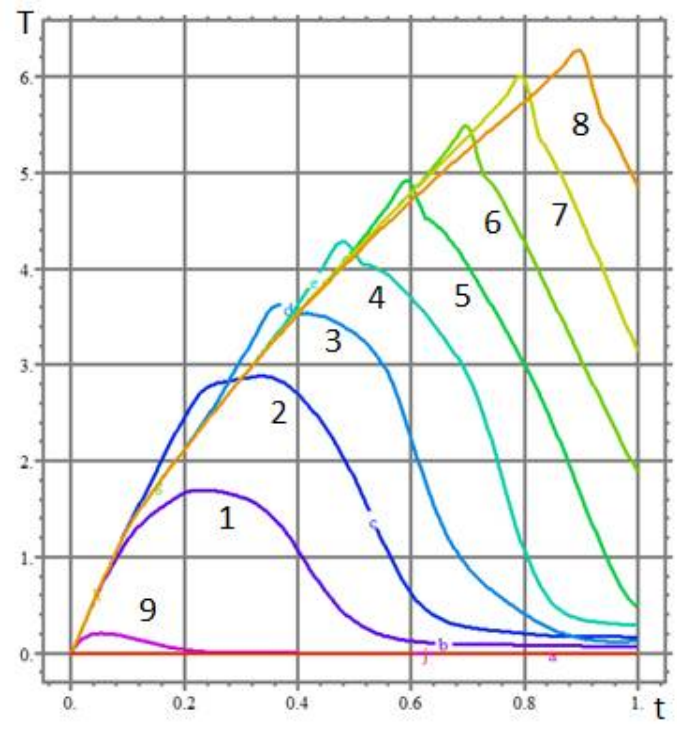

A

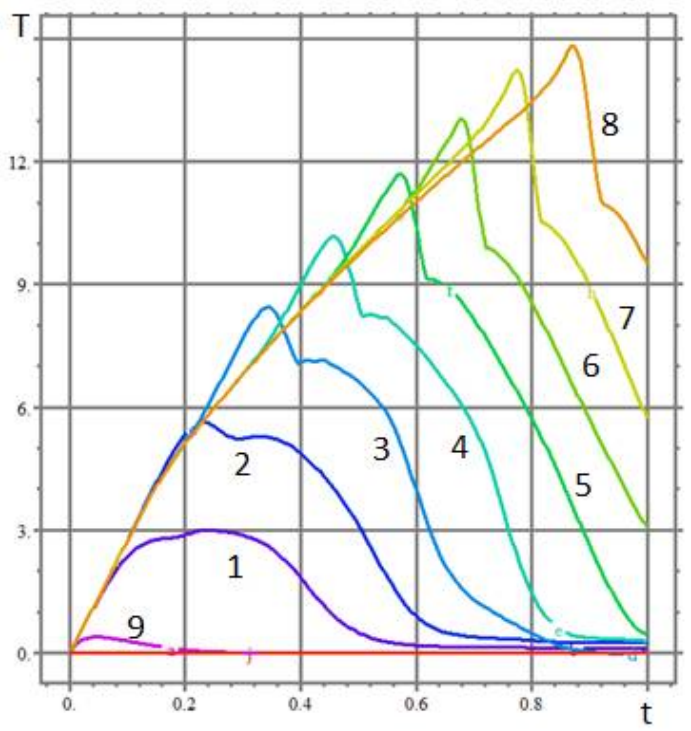

B

Рис. 10. Сравнение динамики температуры твердой фазы $T_{S}\left(t, x, r_{0}\right)$ при начальной пористости $\chi_{0}=0.2$ (А) и $\chi_{0}=0.6(\mathrm{~B})$ в контрольных точках с координатами $(0,0.5),(0.15,0.5),(0.375,0.5)$, $(0.75,0.5),(1.125,0.5),(1.5,0.5),(1.875,0.5),(2.25,0.5)$ представлено кривыми (1-8) соответственно, при концентрационном расширение газовой и твердой фаз (5), (6)
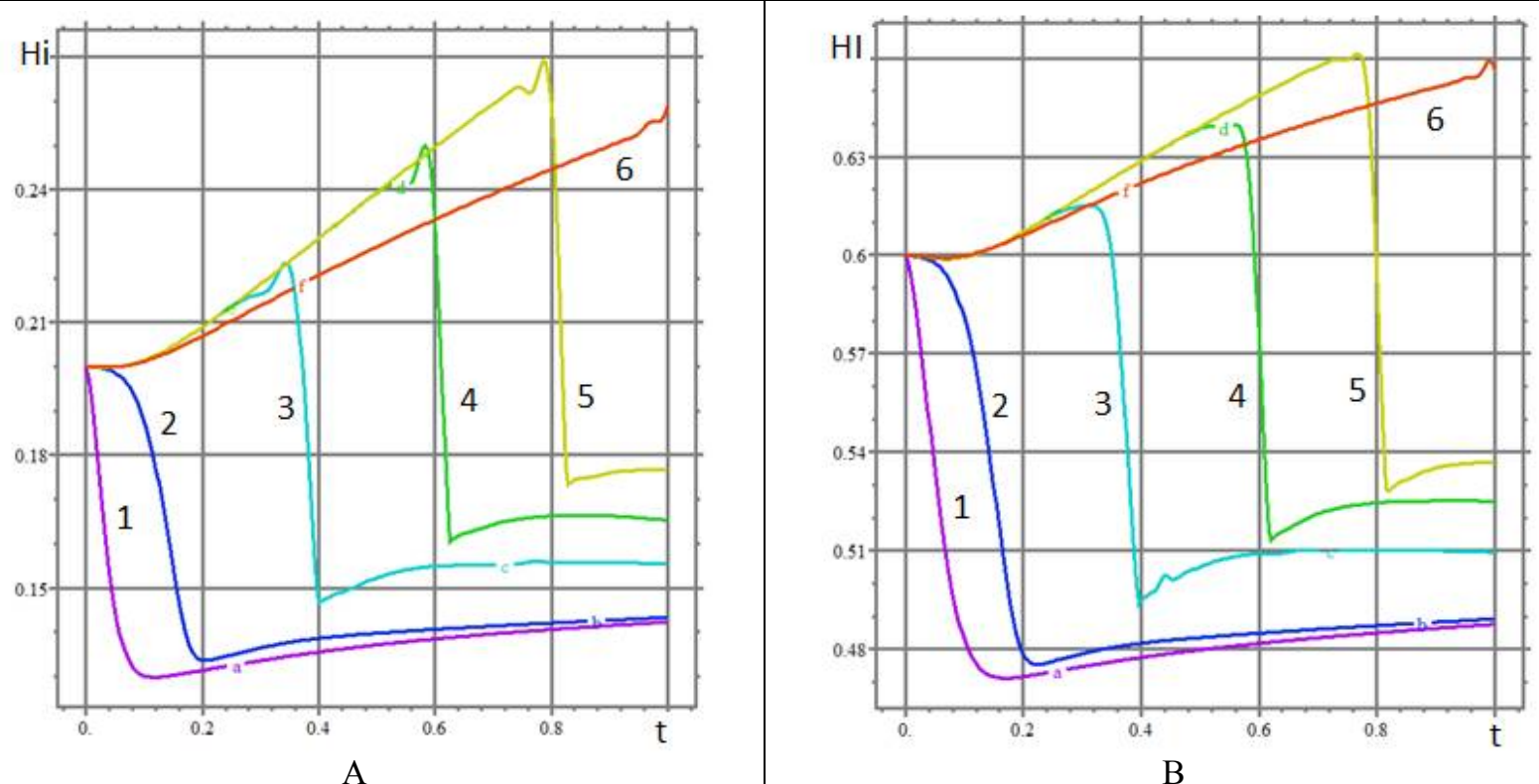

Рис. 11. Концентрационное расширение газовой и твердой фаз. Сравнение динамики пористости при начальной пористости $\chi_{0}=0.2$ (А) и $\chi_{0}=0.6$ (В) в контрольных точках $(x, r)$ с координатами $(0,0.5),(0.15,0.5),(0.75,0.5),(1.5,0.5),(2.25,0.5),(3,0.5)$ иллюстрируется кривыми $(1-6)$ соответственно 
Можно отметить интервалы времени роста пористости, которые сменяются резким уменьшением и последующим повторным возрастанием пористости. Рост пористости вызван воздействием концентрационного расширения газа в то время как уменьшение пористости обусловлено превалирующим эффектом концентрационного и теплового расширения твердой фазы.

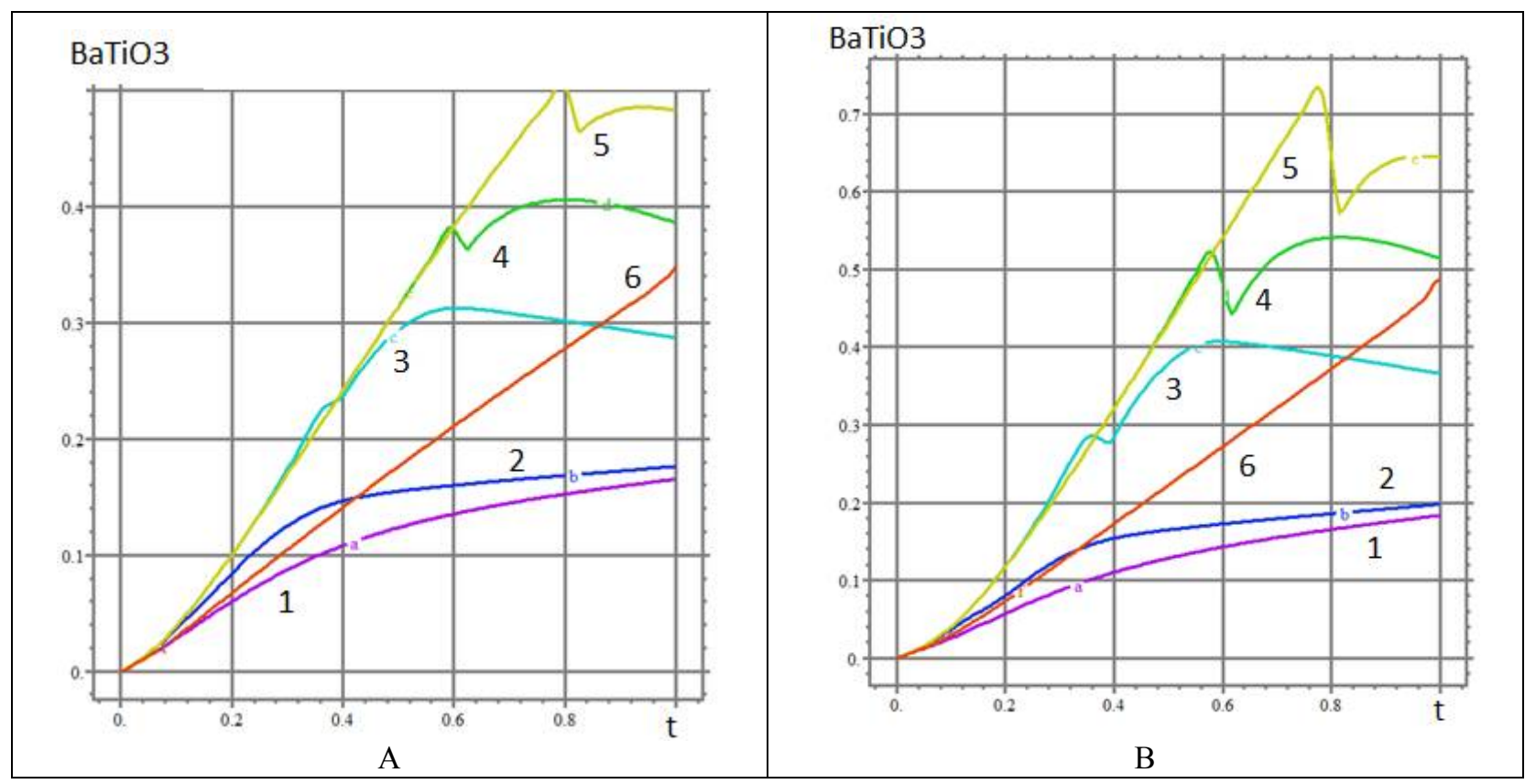

Рис. 12. Концентрационное расширение газовой и твердой фаз. Сравнение динамики синтеза титаната бария при начальной пористости $\chi_{0}=0.2$ (А) и $\chi_{0}=0.6$ (В) в контрольных точках $(x, r)$ с координатами $(0,0.5),(0.15,0.5),(0.75,0.5),(1.5,0.5),(2.25,0.5),(3,0.5)$ иллюстрируется кривыми 1-6 соответственно

Моделирование позволяет утверждать замедление синтеза титаната бария при начальной $\chi_{0}=0.6$ в сравнении с $\chi_{0}=0.2$. Эффект составляет примерно $50 \%$ (кривые 4 и 5).

\section{4. Заключение}

1. Проведено исследование объемных изменений и переменной пористости при концентрационном расширении твердой фаза в синтезе титаната бария в трехзонном реакторе. Предложено обобщение двухтемпературной модели $[14,15]$ и формула переменной пористости при самосогласованном расчете коэффициентов концентрационного расширения, тепловой и массовой дисперсии. Коэффициенты концентрационного расширения находятся в процессе распространения фронта горения в пористой зоне реактора в зависимости от кинетики синтеза титаната бария при заданных начальных величинах плотности и коэффициентов расширения реагентов в газовой и твердой фазе.

2. Оценка объемных деформаций. возникающих вследствие изменения температуры и концентраций смеси реагентов и продуктов синтеза титаната бария методом CCSO, позволяет утверждать что изменение температуры в процессе горения углерода существенно зависит от начального фазового состава и приводят к изменению конечного распределения массы химического состава образца, объема и времени протекания процесса.

3. Проведенное моделирование синтеза указывает на рост температуры обеих фаз с уменьшением пористости. Пористость неравномерно распределена в реакторе. Полученные зависимости скоростей объемного расширения, коэффициента пористости, температур и 
плотностей компонент синтеза от начальных коэффициентов теплового и концентрационного расширения демонстрируют значительное влияние переменной пористости на процесс синтеза мелкодисперсных частиц титаната бария.

Работа выполнена по теме государственного задания № госрегистрацииАААА-А20120011690135-5.

\section{Литература}

1. Martirosyan K. S. and Luss D. Carbon Combustion Synthesis of Oxides Process Demonstration and Features AIChE 2005, vol. J 51 10, pp. 2801-2810.

2. Марков А.А., Обосян М.А., Мартиросян К.С. Исследование синтеза ферритов за волной горения с применением моделей скольжения и скачков температуры и концентраций компонент газовой фазы на поверхности пор твердой фазы. Физико-химическая кинетика в газовой динамике 2015 Vol.16 (1).

3. Марков А.А., Филимонов И.А., Мартиросян К.С. Моделирование синтеза сложных оксидов субмикронной дисперсности. ТОХТ 2017 51, № 1, с. 31-42.

4. Markov A.A., Filimonov I.A., and Martirosyan K.S., Synthesis simulation of submicron particles of complex oxides // Theoretical Foundations of Chemical Engineering 2017(1) pp.1-12.

5. Markov A.A., Filimonov I.A., and Martirosyan K.S. Carbon Combustion Synthesis of Oxides: Effect of Mach, Peclet, and Reynolds Numbers on Gas Dynamics. International Journal of Self Propagating High Temperature Synthesis, 2013, 22, No. 1, pp. 11-17.

6. Markov A.A., Filimonov I.A., and Martirosyan K.S. Simulation of front motion in a reacting condensed two phase mixture, J. Comput. Phys. Volume 231, Issue 20, 15 August 2012, pp. 6714-6724 (2012).

7. Markov A.A., On Thermal and Mass Dispersion Effect on Barium Titanate Synthesis via CCSO. Physical-Chemical Kinetics in Gas Dynamics 2019 V20 (4) pp. 1-14. http://chemphys.edu.ru/issues/201920-4/articles/870/, http://www.chemphys.edu.ru DOI: http://doi.org/10.33257/PhChGD.20.4.870.

8. Марков А.А. О влиянии тепловой и массовой дисперсии на синтез микронных частиц титаната бария. // Вестник ПНИПУ. Химическая технология и биотехнология. 2020. № 2, с. 160-177. DOI: $10.15593 / 2224-9400 / 2020.2 .12$

9. Марков А.А. О тепловой и массовой дисперсии при горении углерода в осесимметричном трехзонном реакторе. // Материалы Тринадцатой Международной конференции по Прикладной математике и механике в аэрокосмической отрасли (NPNJ'2020) 24-31 мая 2020 г. Алушта, Крым. М. Изд-во МАИ, с. 508-510.

10. Markov A.A. On fine particles synthesis using three-zone reactor August 2020. Journal of Physics Conference Series 1611:012047. DOI: 10.1088/1742-6596/1611/1/012047.

11. Whitaker S. Transport equations for multi-phase systems. Chemical Engineering Science, 1973, vol. 28, pp. 139-147.

12. Hsu, C.T., Cheng, P. Thermal dispersion in a porous medium. Int. J. Heat Mass Transf. 1990, 33, 1587-1597.

13. Fatehi M. and Kaviany M. Role of gas-phase reaction and gas-solid thermal nonequilibrium in reverse combustion. Int Heat Mass Transfer. 1997, 11, pp. 2607-20.

14. Oliveira A.A.M. and Kaviany M. Nonequilibrium in the transport of heat and reactants in combustion in porous media. Progress in Energy and Combustion Science, 2001, 27, pp. 523-45.

15. Pereira F.M., Oliveira A.A.M. and Fachini F.F. Theoretical analysis of ultra-lean premixed flames in porous inert media. J. Fluid Mech. 2010, 657, pp. 285-307.

16. Fatehi M. and Kaviany M. Role of gas-phase reaction and gas-solid thermal nonequilibrium in reverse combustion. Int. Heat Mass Transfer. 1997, 11, pp. 2607-2620. 
17. Delgado JMPQ 2007 Longitudinal and transverse dispersion in porous media. ChemEng Res Des, 2007, 85, pp. 1245-1252.

18. Сорокова С.Н., Князева А.Г. Связанная модель спекания порошков системы $\mathrm{Ti}_{-} \mathrm{TiAI}_{3} / /$ Известия Томского политехнического университета. 2009. Т. 314. №2. С. 96-101.

19. Сорокова С.Н., Князева А.Г. Математическое моделирование объемных изменений в процессе спекания порошков системы Ti-Al//Физическая мезомеханика, 11, 6 (2008), с. 95-101.

20. C. Trevino De Leo, G.C. Dannangoda, M.A. Hobosyan, J.T. Held, F. Safi Samghabadi, M. Khodadadi, D. Litvinov, K.A. Mkhoyan, K.S. Martirosyan. Carbon combustion synthesis of Janus-like particles of magnetoelectric cobalt ferrite and barium titanate// Ceramics International, Volume 47, Issue 4, 15 February 2021, pp. 5415-5422. https://doi.org/10.1016/j.ceramint.2020.10.123

21. Brzozowski E., Sanchez J., and Castro M.S. $\mathrm{BaCO}_{3}-\mathrm{TiO}_{2}$ Solid State Reaction: A Kinetic Study. Journal of Materials Synthesis and Processing. 10, 1, 2002, pp. 1064-7562.

22. Beauger A., Mutin J.C., Niepce J.C. Synthesis reaction of metatitanate $\mathrm{BaTi0}_{3}$. Part 1 Effect of the gaseous atmosphere upon the thermal evolution of the system BaCO3-Ti02. Journal of materials science. Vol.18. 1983. Pp. 3041-3046.

23. Chen K., Martirosyan K.S., and Luss D. Hot Zones Formation During Regeneration of Diesel Particulate Filters, AIChE Journal, 57, 2, Feb. 2011, pp. 497-506.

24. Верятин У.Д., Маширев В.П. и др. Термодинамические свойства неорганических веществ: Справочник / Под ред. АП. Зефирова. - М.: Атомиздат, 1965. 460 с.

25. Markov A.A. Jump-Slip simulation technique for combustion in submicron tubes and submicron pores. Computers and Fluids, 99C, 2014, pp. 83-92.

26. Markov A.A., Hobosyan M.A., and Martirosyan K.S. Ferrite Synthesis Simulation via Carbon Combustion using Slip, Temperature, and Concentration Gas Species Jump at Pore Surface// PhysicalChemical Kinetics in Gas Dynamics. 2015. Vol. 16, No. 1. http://chemphys.edu.ru/issues/2015-16$\underline{1 / \text { articles/506/ }}$

27. Markov A.A., Hobosyan M.A., and Martirosyan K.S. Simulation of heat and masstransfer in pores as applied to synthesis of magnesium-zinc and nickel-zinc ferrite nanoparticles// Nanomechanics Science and Technology: An International Journal, 6(3), 2015, pp. 1-14.

28. Kubaschewski O., and Alcock C.B., Metallurgical Thermochemistry, Pergamon press, 1979.

29. Quintard, M., Whitaker, S. Theoretical Analysis of Transport in Porous Media. Marcel Dekker, New York, 2000.

30. Боли Б., Уийиер Дж. Теория температурных напряжений. - М.: Мир. 1964. 518 с.

31. Kolthoum I. Othman1, Ahmed A. Hassan, Omar A. A. Abdelal1, Ezzat S. Elshazly, M. El-Sayed Ali, S. M. El-Raghy, S. El-Houte, Formation Mechanism of Barium Titanate by Solid-State Reactions. International Journal of Scientific \& Engineering Research, Vol. 5, Issue 7, July 2014 ISSN 22295518.

32. Франк-Каменецкий Д. А. Диффузия и теплопередача в химической кинетике Наука (Изд. 3-е, испр. и доп.), 1987, 491 с.

\section{References}

1. Martirosyan, K. S., and Luss, D., "Carbon Combustion Synthesis of Oxides Process Demonstration and Features,” AIChE, Vol. J 51, No. 10, 2005, pp. 2801-2810.

2. Markov, A. A., Hobosyan, M. A., Martirosyan, K. S., "Ferrite Synthesis Simulation via Carbon Combustion using Slip, Temperature, and Concentration Gas Species Jump at Pore Surface," PhysicalChemical Kinetics in Gas Dynamics, Vol. 16, No. 1, 2015. http://chemphys.edu.ru/issues/2015-161/articles/506/

3. Markov, A. A., Filimonov, I. A., Martirosjan, K. S., "Modelirovanie sinteza slozhnyh oksidov submikronnoj dispersnosti." TOHT, Vol. 51, No. 1, 2017, pp. 31-42. 
4. Markov, A. A., Filimonov, I. A., and Martirosyan, K. S., "Synthesis simulation of submicron particles of complex oxides," Theoretical Foundations of Chemical Engineering, Vol. 1, 2017, pp.1-12.

5. Markov, A. A., Filimonov, I. A., and Martirosyan, K. S., "Carbon Combustion Synthesis of Oxides: Effect of Mach, Peclet, and Reynolds Numbers on Gas Dynamics," International Journal of Self Propagating High Temperature Synthesis, Vol. 22, No. 1, 2013, pp. 11-17.

6. Markov, A. A., Filimonov, I. A., and Martirosyan, K. S., "Simulation of front motion in a reacting condensed two phase mixture," J. Comput. Phys., Vol. 231, Issue 20, Aug. 2012, pp. 6714-6724.

7. Markov, A. A., "On Thermal and Mass Dispersion Effect on Barium Titanate Synthesis via CCSO," Physical-Chemical Kinetics in Gas Dynamics, Vol. 20, No. 4, 2019. http://chemphys.edu.ru/issues/2019-20-4/articles/870/ DOI: 10.33257/PhChGD.20.4.870

8. Markov, A. A., "O vlijanii teplovoj i massovoj dispersii na sintez mikronnyh chastic titanata barija," Vestnik PNIPU. Himicheskaja tehnologija i biotehnologija, No. 2, 2020, pp. 160-177.

DOI: 10.15593/2224-9400/2020.2.12

9. Markov, A. A., "O teplovoj i massovoj dispersii pri gorenii ugleroda v osesimmetrichnom trehzonnom reaktore," Materialy Trinadcatoj Mezhdunarodnoj konferencii po Prikladnoj matematike i mehanike v ajerokosmicheskoj otrasli (NPNJ'2020), May 2020, Alushta, Krym. M. Izd-vo MAI, pp. 508-510.

10. Markov, A. A., "On fine particles synthesis using three-zone reactor," Journal of Physics Conference Series 1611:012047, Aug. 2020. DOI: 10.1088/1742-6596/1611/1/012047

11. Whitaker, S., "Transport equations for multi-phase systems," Chemical Engineering Science, Vol. 28, 1973, pp. 139-147.

12. Hsu, C. T., Cheng, P., "Thermal dispersion in a porous medium," Int. J. Heat Mass Transf., Vol. 33, 1990, pp. 1587-1597.

13. Fatehi, M., and Kaviany, M., "Role of gas-phase reaction and gas-solid thermal nonequilibrium in reverse combustion," Int. Heat Mass Transfer, Vol. 11, 1997, pp. 2607-20.

14. Oliveira, A. A. M., and Kaviany, M., "Nonequilibrium in the transport of heat and reactants in combustion in porous media," Progress in Energy and Combustion Science, Vol. 27, 2001, pp. 523-45.

15. Pereira, F. M., Oliveira, A. A. M., and Fachini, F. F., "Theoretical analysis of ultra-lean premixed flames in porous inert media," J. Fluid Mech., Vol. 657, 2010, pp. 285-307.

16. Fatehi, M., and Kaviany, M., "Role of gas-phase reaction and gas-solid thermal nonequilibrium in reverse combustion," Int. Heat Mass Transfer, 11, 1997, pp. 2607-2620.

17. Delgado, J. M. P. Q., "Longitudinal and transverse dispersion in porous media," Chem. Eng. Res. Des., Vol. 85, No. 9, 2007, pp. 1245-1252.

18. Sorokova, S. N., Knjazeva, A. G., "Svjazannaja model' spekanija poroshkov sistemy Ti-TiAI3," Izvestija Tomskogo politehnicheskogo universiteta, Vol. 314, No. 2, 2009, pp. 96-101.

19. Sorokova, S. N., Knjazeva, A. G., "Matematicheskoe modelirovanie ob\#emnyh izmenenij v processe spekanija poroshkov sistemy Ti-Al," Fizicheskaja mezomehanika, Vol. 11, No. 6, 2008, pp. 95-101.

20. De Leo, Trevino, Dannangoda, G. C., Hobosyan, M. A., Held, J. T., Samghabadi, F. Safi, Khodadadi, M., Litvinov, D., Mkhoyan, K. A., Martirosyan, K. S., "Carbon combustion synthesis of Janus-like particles of magnetoelectric cobalt ferrite and barium titanate," Ceramics International, October 2020. https://doi.org/10.1016/j.ceramint.2020.10.123

21. Brzozowski, E., Sanchez, J., and Castro, M. S., "BaCO $3-\mathrm{TiO}_{2}$ Solid State Reaction: A Kinetic Study," Journal of Materials Synthesis and Processing, Vol. 10, No. 1, 2002, pp. 1064-7562.

22. Beauger, A., Mutin, J. C., Niepce, J. C., "Synthesis reaction of metatitanate BaTi0 3 . Part 1. Effect of the gaseous atmosphere upon the thermal evolution of the system $\mathrm{BaCO}_{-}-\mathrm{Ti} 0_{2}$, " Journal of materials science, Vol. 18, 1983, pp. 3041-3046.

23. Chen, K., Martirosyan, K. S., and Luss, D., "Hot Zones Formation During Regeneration of Diesel Particulate Filters," AIChE Journal, Vol. 57, No. 2, Feb. 2011, pp. 497-506. 
24. Verjatin, U. D., Mashirev, V. P. i dr., Termodinamicheskie svojstva neorganicheskih veshhestv. Spravochnik. Pod red. AP. Zefirova (Thermodynamic properties of inorganic substances. Handbook. Ed. by AP. Zefirov), M.: Atomizdat, 1965. 460 p.

25. Markov, A. A., "Jump-Slip simulation technique for combustion in submicron tubes and submicron pores," Computers and Fluids, Vol. 99C, 2014, pp. 83-92.

26. Markov, A. A., Hobosyan, M. A., and Martirosyan, K. S., "Ferrite Synthesis Simulation via Carbon Combustion using Slip, Temperature, and Concentration Gas Species Jump at Pore Surface," PhysicalChemical Kinetics in Gas Dynamics, Vol. 16, No. 1. 2015. http://chemphys.edu.ru/issues/2015-16$1 /$ articles/506/

27. Markov, A. A., Hobosyan, M. A., and Martirosyan, K. S., "Simulation of heat and masstransfer in pores as applied to synthesis of magnesium-zinc and nickel-zinc ferrite nanoparticles," Nanomechanics Science and Technology: An International Journal, Vol. 6, No. 3, 2015, pp. 1-14.

28. Kubaschewski, O., and Alcock, C. B., Metallurgical Thermochemistry, Pergamon press, 1979.

29. Quintard, M., Whitaker, S. Theoretical Analysis of Transport in Porous Media. Marcel Dekker, New York, 2000.

30. Boli, B., Uijier, Dzh., Teorija temperaturnyh naprjazhenij (Temperature tensions theory). M.: Mir, $1964,518 \mathrm{p}$.

31. Kolthoum I., Othman, Ahmed A., Hassan, Omar A. A., Abdeal, Ezzat S., Elshazly, M. El-Sayed, Ali, S. M., El-Raghy, S. El-Houte, "Formation Mechanism of Barium Titanate by Solid-State Reactions," International Journal of Scientific \& Engineering Research, Vol. 5, Issue 7, July-2014. ISSN 22295518

32. Frank-Kameneckij, D. A. Diffuzija i teploperedacha $v$ himicheskoj kinetike (Diffusion and heat transfer in chemical kinetics), Nauka (Izd. 3-e, ispr. i dop.), 1987, $491 \mathrm{c.}$

\section{Список обозначений}

$\mathrm{BaO}$
$\mathrm{BaTiO}_{3}, b_{1}$
$c_{p}, c_{p}=C_{p, \text { air }}$
$\tilde{c}_{S}, \tilde{c}_{p g}, \tilde{c}_{S}=C_{S} / c_{p}$,
$\tilde{c}_{p g}=C_{p g} / c_{p}$
$C_{j}, j=1,2,3$
$B_{\mathrm{jg}}=\frac{Y_{\mathrm{jg}}}{Y_{\mathrm{g}, \mathrm{tot}}}, B_{j S}=\frac{Y_{j S}}{Y_{S, t o t}}$
$D_{0}$
$\tilde{D}_{,}, \tilde{D}=D / D_{0}$
$\mathrm{D}_{m g}$
$D_{L}, D_{T r}$
$d_{p}$

Оксид бария

Титанат бария

Константы зависимости тензоров дисперсии от локального числа Пекле (безразмерные)

Характерная теплоемкость газовой фазы при постоянном давлении $(\mathrm{J} / \mathrm{kg} / \mathrm{K})$

Безразмерные теплоемкости твердой и газовой фазы

\section{Массовые доли газовых компонент}

Молярные доли (концентрации) газовых компонент и компонент твердой фазы

Характерная величина коэффициента диффузии

$D_{0}=2 \times 10^{-5},\left(\mathrm{~m}^{2} / \mathrm{s}\right)$

Безразмерный коэффициент диффузии

Дисперсионный тензор диффузии массы $\left(\mathrm{m}^{2} / \mathrm{s}\right)$

Компоненты продольной и поперечной дисперсии массы $\left(\mathrm{m}^{2} / \mathrm{s}\right)$

Диаметр частиц (m) 


\begin{tabular}{|c|c|}
\hline $\mathrm{D}_{\mathrm{Tg}}$ & Дисперсионный тензор диффузии тепла $\left(\mathrm{W} \cdot \mathrm{m}^{-1} \cdot \mathrm{K}^{-1}\right)$ \\
\hline E & Энергия активации $\left(\mathrm{J} \cdot \mathrm{mole}^{-1}\right)$ \\
\hline I & Единичный тензор \\
\hline$J_{i S}, i=1, \ldots 5$ & Массовые потоки синтеза $\mathrm{BaTiO}_{3}\left(\mathrm{~kg} \cdot \mathrm{m}^{-2} \cdot \mathrm{s}^{-1}\right)$ \\
\hline$k$ & Частотный фактор в формуле Аррениуса $\left(\mathrm{s}^{-1}\right)$ \\
\hline $\begin{array}{l}K \\
l_{0}\end{array}$ & $\begin{array}{l}\text { Изотермический модуль всестороннего сжатия } \\
\text { Характерный размер (m) }\end{array}$ \\
\hline $\mathrm{Ma}$ & Число Маха (безразмерное) \\
\hline$M_{0}$ & Характерная молярная масса $\left(\mathrm{kg} \cdot \mathrm{mole}^{-1}\right)$ \\
\hline$M_{1 g}, M_{2 g}, M_{S}$ & Молярные массы газовых компонент $\left(\mathrm{kg} \cdot \mathrm{mole}^{-1}\right)$ \\
\hline$M_{i S}, i=1, \ldots 5$ & Молярные массы компонент синтеза $\mathrm{BaTiO}_{3},\left(\mathrm{~kg} \cdot \mathrm{mole}^{-1}\right)$ \\
\hline$p_{0}$ & Характерное давление $R \rho_{0} T M_{0}^{-1},(\mathrm{~Pa})$ \\
\hline$p_{g}$ & Давление газа (Pa) \\
\hline $\mathrm{Pe}_{1}$ & Диффузионное число Пекле $\mathrm{Pe}_{1}=l_{0}^{2} / t_{0} D_{0}$ (безразмерное) \\
\hline & $\begin{array}{l}\text { Локальные числа Пекле для продольной и поперечной дисперсии } \\
\text { тепла, (безразмерные) }\end{array}$ \\
\hline $\mathrm{Pe}_{1 t}, \mathrm{Pe}_{2 t}$ & $\mathrm{Pe}_{1 t}=\frac{\chi|u| d_{p}}{2 a_{g}}, \mathrm{Pe}_{2 t}=\frac{\chi|v| d_{p}}{2 a_{g}}$ \\
\hline $\mathrm{Pe}_{T g}, \mathrm{Pe}_{T s}$ & $\begin{array}{l}\text { Тепловые числа Пекле для газовой и твердой фазы, (безразмер- } \\
\text { ные) }\end{array}$ \\
\hline$Q$ & Тепловой эффект химической реакции (J/kg) \\
\hline$Q_{r}$ & Теплота химической реакции $\left(\mathrm{W} \cdot \mathrm{m}^{-2}\right)$ \\
\hline $\mathrm{Re}$ & Число Рейнольдса (безразмерное) \\
\hline $\mathrm{R}_{\text {solid }}$ & число Рейнольдса твердой фазы (безразмерное) \\
\hline $\mathrm{Re}_{l o c}, \mathrm{Pe}_{T l o c}$ & $\begin{array}{l}\text { Локальные числа Рейнольдса и Пекле (безразмерные) } \\
\operatorname{Re}_{l o c}=\operatorname{Re}|\tilde{\mathbf{u}}| \tilde{\rho}_{g}, \operatorname{Pe}_{T l o c}=\operatorname{Pe}_{T g}|\tilde{\mathbf{u}}| \tilde{\rho}_{g}\end{array}$ \\
\hline $\mathbf{S}_{\mathbf{V}}$ & Слагаемое распределенного сопротивления газа в порах $(\mathrm{Pa} / \mathrm{m})$ \\
\hline$t_{0}$ & Характерное время (s) \\
\hline$t$ & Время (s) \\
\hline$\tilde{t}$ & Безразмерное время $\tilde{t}=t / t_{0}$ \\
\hline$V$ & Удельный объем $\left(\mathrm{m}^{3}\right)$ \\
\hline$V_{i, \text { solid }}$ & Компоненты скорости твердой фазы \\
\hline$\frac{\rho_{\mathrm{lg}}}{M_{\mathrm{lg}}}, \quad Y_{\mathrm{g}, \mathrm{tot}}=\sum_{l=1}^{3} Y_{\mathrm{lg}}$ & $\begin{array}{l}\text { Молярные плотности компонент газовой фазы }\left(\mathrm{mole} / \mathrm{m}^{3}\right) \text {, сум- } \\
\text { марная плотность газа }\end{array}$ \\
\hline$Y_{j S}=\frac{\rho_{j S}}{M_{j S}}$ & Молярные плотности компонент твердой фазы $\left(\mathrm{mole} / \mathrm{m}^{3}\right)$ \\
\hline$Y_{S, t o t}=\sum_{j=1} Y_{j S}$ & Суммарная молярная плотность твердой фазы \\
\hline
\end{tabular}




\section{Греческие буквы}

$$
\begin{gathered}
a_{g} \\
\eta_{j}, \eta_{1 j}, \eta_{0 j}, \quad j=1,2,3 \\
\eta_{j}=\eta_{1 j}|\mathrm{u}|+\eta_{0 j}
\end{gathered}
$$

$$
\bar{\alpha}_{i g}, \bar{\alpha}_{i S}
$$

$\rho_{1 g}=\rho_{\mathrm{O}_{2}}, \rho_{2 g}=\rho_{\mathrm{CO}_{2}}$

$\rho_{3 g}=\rho_{\mathrm{N}_{2}}$

$$
\rho_{g}=\rho_{1 g}+\rho_{2 g}+\rho_{3 g}
$$$$
\rho_{1 S}=\rho_{\mathrm{C}}, \rho_{2 S}=\rho_{\mathrm{BaCO}_{3}} \text {, }
$$$$
\rho_{4 S}=\rho_{\mathrm{TiO}_{2}}, \rho_{5 S}=\rho_{\mathrm{BaTiO}_{3}},
$$$$
\rho_{6 S}=\rho_{\mathrm{Ba}_{2} \mathrm{TiO}_{4}}
$$

$$
\begin{aligned}
& \rho_{S}=\sum_{j=1}^{6} \rho_{j S} \\
& \rho_{t o t}=\rho_{\mathrm{g}}+\rho_{S} \\
& \sigma_{i j}
\end{aligned}
$$$$
\sigma_{i j}=2 \mu_{S 1} \varepsilon_{i j}+\delta_{i j}\left(\mu_{S 2} \sum_{k} \varepsilon_{k k}-K \omega\right)
$$

Суммарная плотность компонент твердой фазы

$$
\text { Суммарная массовая плотность }
$$

Тензор напряжений в твердой фазе (Рa)

$$
\begin{aligned}
& \eta_{j}, j=1,2,3 \\
& \lambda_{0} \\
& \tilde{\lambda}_{g}, \tilde{\lambda}_{S} \\
& \mu \\
& \mu_{S 1}, \mu_{S 2}, \mu_{S} \\
& v_{\mathrm{lg}}=\frac{M_{\mathrm{lg}}}{\rho_{\mathrm{lg}}} \\
& v_{j S}=\frac{M_{j S}}{\rho_{j S}} \\
& \varsigma_{j}, j=1,2,3
\end{aligned}
$$

Компоненты распределенного сопротивления пористости $(\mathrm{Pa} / \mathrm{m})$,

$$
\eta_{j}=\alpha_{j}|\mathrm{u}|+\varsigma_{j}
$$

Характерная величина коэффициента теплопроводности

$$
\left(\mathrm{W} \cdot \mathrm{m}^{-1} \cdot \mathrm{K}^{-1}\right), \lambda_{0}=\lambda_{\text {air }}=0.06
$$

Безразмерные коэффициенты теплопроводности газовой и твердой фаз $\tilde{\lambda}_{g}=\lambda_{\text {air }} / \lambda_{0}, \tilde{\lambda}_{S}=\lambda_{S} / \lambda_{0}$

Коэффициент динамической вязкости газа $\left(\mathrm{kg} \cdot \mathrm{m}^{-1} \cdot \mathrm{s}^{-1}\right)$

Модули упругости (коэффициенты Ламе) твердой фазы (Рa),

$$
\left(2 \mu_{S 1}+3 \mu_{S 2}\right)=3 \mu_{S}
$$

Молярный объем компонента газовой фазы

\section{Молярный объем компонента твердой фазы}

Компоненты распределенного сопротивления пористости $(\mathrm{Pa} / \mathrm{m})$,

$$
\eta_{j}=\alpha_{j}|\mathrm{u}|+\varsigma_{j}
$$


$\kappa$

$\tilde{\kappa}=\kappa \mathrm{t}_{0} \mathrm{~A} /\left(c_{p} \rho_{0} V\right)$

$\kappa_{0}$

$\chi$

$\chi_{0}$

$\omega_{g}, \omega_{S}$

$\omega_{T}$

$\omega=\omega_{T}+\omega_{S}$
Коэффициент теплообмена $\left(\mathrm{J} \cdot \mathrm{K}^{-1} \cdot \mathrm{m}^{-2} \cdot \mathrm{s}^{-1}\right)$

Безразмерный коэффициент межфазового теплообмена

Размерный коэффициент в формуле Левека

$\kappa=\kappa_{0}\left(1+\operatorname{Re}_{\text {loc }}^{0.3} P e_{\text {Tloc }}^{0.3}\right)$

Коэффициент пористости (безразмерный)

Начальная пористость, $\chi_{0}=V_{g, 0} / V_{0}$

Коэффициенты концентрационного расширения (безразмерные) линейный коэффициент теплового расширения, (безразмерный), $\omega_{T}=3 \alpha_{T}\left[\left(-T_{g} / T_{g}^{0}\right)+1\right]$

Суммарный коэффициент объемного расширения твердой фазы 